DOE/ER/41018-8

\title{
EXPERIMENTS ON THE NUCLEAR INTERACTIONS OF PIONS AND ELECTRONS
}

\author{
Closeout Performance Report
}

for Period Aug. 15, 2003-April 1, 2005

\author{
R. C. Minehart \\ Department of Physics \\ University of Virginia \\ 205 McCormick Road \\ Charlottesville, VA 22901
}

April 15, 2005

Prepared for

THE U.S. DEPARTMENT OF ENERGY

AWARD NUMBER DE-FG02-97ER41018 


\section{SUMMARY}

This is the final technical report. Yearly Progress Reports were submitted throughtout the duration of the project. Along with our publications, these reports provide a detailed record of our accomplishments. This report largely consists of a summary of the technical activities carried out during last 2-1/2 years of the project. Our work during these years centered primarily around experiments using the CLAS detector at the Thomas Jefferson Laboratory (JLab), for which R. Minehart was a co-spokesman.

The following is a summary of the projects supported by our DOE grant:

1. Electro-production of excited states of the nucleon through the analysis of exclusive single pion production reactions induced by polarized electrons incident on both polarized and unpolarized nucleon targets. The experimental measurements were made at JLab.

2. Measurement of proton and deuteron spin structure functions in and above the nucleon resonance region at low and moderate $Q^{2}$, using inclusive $\vec{e} \vec{p}$ and $\vec{e} \vec{d}$ scattering. The measurements were made at JLab.

3. A precise measurement of the branching ratio for pion beta decay was carried out along with other members of the PIBETA collaboration. The measurements were made at the PSI Laboratory in Switzerland. The technical aspects of the detector constructed for this project was published in Nuclear Instruments and Methods. The scientific results were published in articles in the Physical Review Letters.

4. The PRIMEX experiment at JLab used the CLAS bremsstrahlung tagger along with its own specialized detector. W. Stephens from our group designed and built specialized electronics for the gamma-ray shower detector. The data for this experiment were taken in the fall of 2004, and the data are still being analyzied. (Note: First results of the Primakov measurements were reported two years after the end of this grant, at American Physical Society meetings in the spring of 2007)

We have reported on our research at several international meetings. Papers published in the period from 2002-2005 are listed according to one of the above topics in the section on publications. This section also includes a list of other papers produced by the CLAS collaboration. 


\section{Contents}

1 The $\mathbf{N}^{*}$ Program $\quad 6$

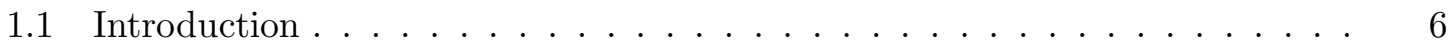

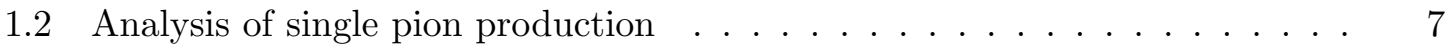

1.3 Experimental Results . . . . . . . . . . . . . . . . . . . 8

$1.3 .1 \quad \mathrm{E} 89-037 \ldots \ldots \ldots \ldots$

$1.3 .2 \quad \mathbf{E 8 9 - 0 4 2} \ldots \ldots \ldots \ldots$

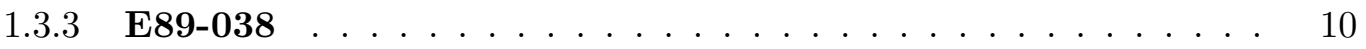

1.3.4 Polarization observables . . . . . . . . . . . . . . . . . . 11

$1.4 N^{*}$ Low Energy Experiments . . . . . . . . . . . . . . . . . 11

2 EG1a and EG1b 18

2.1 The Physics of Double Polarization Experiments . . . . . . . . . . . 18

2.2 CLAS Experiments . . . . . . . . . . . . . . . . . . 20

2.3 Determination of Inclusive Structure Functions . . . . . . . . . . . . . . 22

$3 \quad$ PRIMEX Experiment $\quad 37$

4 A PRECISE MEASUREMENT OF THE $\pi^{+} \rightarrow \pi^{0} e^{+} \nu$ DECAY RATE 37

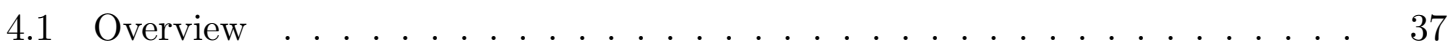

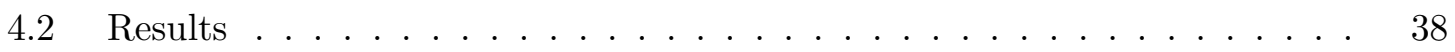

5 Publications and Talks 41

5.1 Published Papers: . . . . . . . . . . . . . . . . . . . . . . . 41

5.2 Talks given at Meetings: . . . . . . . . . . . . . . . . . . 44 


\section{List of Figures}

1 CLAS measurements of $\sigma_{L T^{\prime}}$ versus $\cos \theta_{\pi}^{*}$ for the $\pi^{0} p$ channel (top) the $\pi^{+} n$ channel (bottom) extracted at $Q^{2}=0.40 \mathrm{GeV}^{2}$. Curves show model predictions. Shaded bars show systematic errors . . . . . . . . . . .

$2 \quad Q^{2}$ dependence of Legendre moments of $\sigma_{L T^{\prime}}$ for $\pi^{0} p$ channel (left) and $\pi^{+} n$ channel (right). Curves show model predictions. Data points are the present CLAS measurement. Statistical error only. . . . . . . . . . . . . .

3 Beam asymmetry $A_{L T^{\prime}}$ for the $e p \rightarrow e^{\prime} \pi^{+} n$ reaction. The $W$ dependence is plotted for various bins in $\phi_{\pi}^{*}$. Blue curve show the prediction of MAID with the default Roper strength and with the Roper strength increased by a factor of three (dashed curve) in the $S_{1-}$ longitudinal multipole. Red curve shows the fit of Aznauryan, which also requires a non-zero longitudinal Roper strength. . . . . . . . . . . . . . . . . .

4 CLAS measurements of $\sigma_{L T^{\prime}}$ versus $\cos \theta_{\pi}^{*}$ for the $\pi^{0} p$ channel [3] (top) and for the $\pi^{+} n$ channel [4](bottom) extracted at $Q^{2}=0.40 \mathrm{GeV}^{2}$ and $W=$ $1.18-1.26 \mathrm{GeV}$. The curves show model predictions. The shaded bars show estimated systematic errors. . . . . . . . . . . . . . . . . .

5 Sensitivity of $\sigma_{L T}^{\prime}\left(\pi^{+} n\right)$ to changes in Roper multipoles $M_{1-}$ and $S_{1-}$ using unitary isobar model of Aznauryan [6]. Solid line shows best fit. . . . . . .

$6 \quad Q^{2}$ dependence of transverse $A_{1 / 2}^{p}$ and longitudinal $S_{1 / 2}^{p}$ helicity amplitudes for the Roper $P_{11}(1440)$. Curves show recent quark model calculations. . .

$7 \quad Q^{2}$ dependence of longitudinal $S_{1 / 2}^{p}$ helicity amplitudes for the $S_{11}(1535)$ and $D_{13}(1520)$ resonances from global analysis of CLAS $\pi^{+}$and $\pi^{0}$ electroproduction data. Curves show recent quark model calculations. . . . . . . . . .

8 Preliminary measurements of $p\left(e, e^{\prime} p\right) \pi^{0}$ at $Q^{2}=0.2 \mathrm{GeV}^{2}$ from 2002 CLAS E1E run. Plot at left shows angle $\phi_{\pi^{0}}^{*}$ of $p \pi^{0}$ hadronic decay plane relative to $\left(e, e^{\prime}\right)$ plane. Plot at right shows $p \pi^{0}$ center-of-mass angle $\cos \left(\theta_{\pi^{0}}^{*}\right) . \quad \ldots$

9 Eg1a measurements of $g_{1}(x)$ vs. $\mathrm{x}$ for the proton, for five $\mathrm{Q}^{2}$ bins. The solid line is a calculation using the parametrization described in the text. Double bars indicate statistical and (statistical + systematic) errors. . . . . . . .

10 Eg1a measurements of $\Gamma_{1}$ vs. $Q^{2}$ for the proton. Double bars indicate statistical and (statistical + systematic) errors. (a) Solid circles show the integration up to $\mathrm{W}=2 \mathrm{GeV}$, using only our measurements. Data from SLAC are shown with open circles. The upper dashed line is a calculation using the AO program incorporating resonance production with $\mathrm{W}<2.0 \mathrm{GeV}$. The lower dashed line is a calculation using the MAID model for single meson production only. (b) The inclusive scattering model is used to extend the integral to very low $x$. Solid points are the same as in (a), the open circles include the integration to low $x$. Also shown are two points from SLAC $\left(\mathrm{Q}^{2}<=1.5\right)$. See the text for an explanation of the curves. . . . . . . .

$11 g_{1}(x)$ vs. $\mathrm{x}$ for the proton, for $\mathrm{E}=1.6 \mathrm{GeV}$ (EG1b). The solid line is a calculation using the parametrization described in the text. Errors are statistical

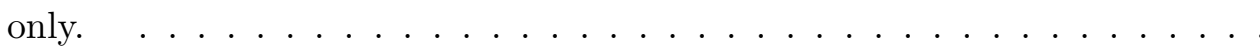


$12 g_{1}(x)$ vs. $\mathrm{x}$ for the proton, for $\mathrm{E}=5.7 \mathrm{GeV}$ (EG1b). The solid line is a calculation using the parametrization described in the text. Errors are statistical

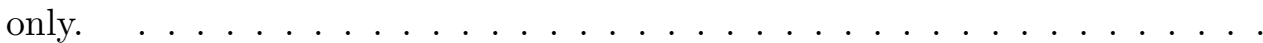

$13 g_{1}(x)$ vs. $\mathrm{x}$ for the deuteron, for $\mathrm{E}=1.6 \mathrm{GeV}$ (EG1b). The solid line is a calculation using the parametrization described in the text. Errors are statistical only. . . . . . . . . . . . . . . . .

$14 g_{1}(x)$ vs. $\mathrm{x}$ for the deuteron, for $\mathrm{E}=5.7 \mathrm{GeV}$ (EG1b). The solid line is a calculation using the parametrization described in the text. Errors are statistical only. . . . . . . . . . . . . . . . . . . 29

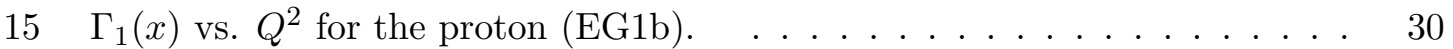

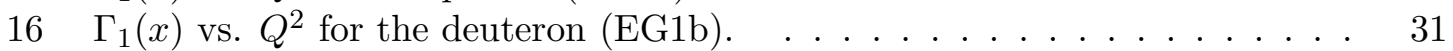

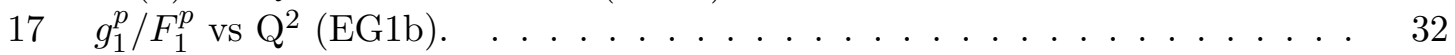

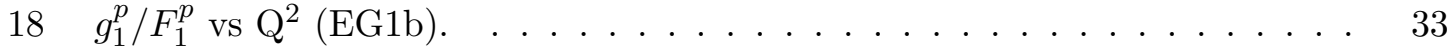

$19 g_{1}^{p}$ Plotted as a function of the Nachtmann variable $\xi$. The red line shows $g_{1}$ evolved from DIS experiments to $\mathrm{Q}^{2}=10 \mathrm{GeV}^{2}$. . . . . . . . . . . . 34

$20 A_{1}^{p}$ vs. $x_{B j}$. High $\mathrm{Q}^{2}$ CLAS data for $W>1.8 \mathrm{GeV}$ is shown along with data from SMC and SLAC Exp. E143. Parameterizations of the world data and predictions of some representative models are shown. . . . . . . . . . .

$21 A_{1}^{d}$ vs. $x_{B j}$. High $\mathrm{Q}^{2}$ CLAS data for $W>1.8 \mathrm{GeV}$ is shown along with data from SMC and SLAC Exp. E143. Parameterizations of the world data and predictions of some representative models are shown. . . . . . . . . . . 


\section{The $\mathrm{N}^{*}$ Program}

\subsection{Introduction}

The extensive set of $\mathrm{N}^{*}$ experiments at JLab was a motivating factor in the design of the CEBAF Large Acceptance Spectrometer (CLAS) in Hall B. The experiments employed both unpolarized and polarized beams and targets to study the electromagnetic transition form factors of baryon resonances through single pion electroproduction on protons and neutrons. Such measurements provide stringent tests of valence quark models and can shed light on quark confinement by probing distance scales covering the transition from perturbative to non-perturbative QCD. With its large acceptance for detection of charged hadrons, the CLAS is sensitive to most of the phase space occupied by the resonance decay products. This program has provided a very large data set, and analysis of various aspects of the data still continue

The use of both proton and deuteron targets allows us to obtain more complete isospin information by combining data from the reactions

$$
\begin{aligned}
& e+p \rightarrow e^{\prime}+p^{\prime}+\pi^{0} \\
& e+p \rightarrow e^{\prime}+n+\pi^{+}
\end{aligned}
$$

and

$$
e+p \rightarrow e^{\prime}+p^{\prime}+\pi^{-}
$$

The development of high polarization electron beams at JLAB, permitted most of the measurements to be made with polarized beams $(P \approx 70 \%)$, which allowed access to observables previously unexplored. Experiments with unpolarized targets (liquid $\mathrm{H}_{2}$ and $\mathrm{D}_{2}$ ) study spin independent and beam-spin dependent structure functions. Experiments with polarized targets (frozen $\mathrm{NH}_{3}$ and $\mathrm{ND}_{3}$ ), measure double-spin dependent terms.

The cross sections are very sensitive to both the spatial and spin structure of the quarks in the nucleon and the nucleon resonances. When the dependence on the helicity of the electron and target nucleon is taken into account the cross sections as bilinear combinations of six independent complex helicity amplitudes. A model-independent determination therefore requires at least 11 different measurements at each value of $\mathrm{Q}^{2}, W$, and $\theta^{*}$. Since many of the amplitudes are small, an experimentally determined model independent characterization is not likely in the foreseeable future. However, with the help of models, considerable progress has been made in our understanding of the resonance structures and the transition processes.

With an unpolarized beam and target the single meson cross section has the form

$$
\sigma=\sigma_{T}+\epsilon \sigma_{L}+\epsilon \sigma_{T T} \cos 2 \phi+\sqrt{\frac{\epsilon(1+\epsilon)}{2}} \sigma_{L T} \cos \phi
$$

In this formula, the polarization factor of the virtual photon, $\epsilon$, is given by

$$
\epsilon=\left[1+2\left(1+\frac{\nu^{2}}{Q^{2}}\right) \tan ^{2}(\theta / 2)\right]^{-1}
$$


where $\nu$ is the energy loss of the scattered electron, $\theta$ is the electron scattering angle, and $\phi$ is the angle that the $\pi-N$ plane is rotated from the electron scattering plane around the $\vec{q}$ direction. By studying the dependence on $\epsilon$ and $\phi$ (keeping $\mathrm{Q}^{2}$ and $W$ fixed) the four terms can be separated. With a polarized beam and target, seven additional terms dependent on the choice of polarization can be isolated. More observables can be obtained by studying the dependence of the cross section on the polarization of the recoil proton.

Theoretical models can be used to separate the contributions of resonances and Born terms to individual partial waves. Determination of the $\mathrm{Q}^{2}$ dependence of the transition form factor for an individual resonance provides a strong constraint on the models used to describe the structure of the resonance and its coupling to the photon.

\subsection{Analysis of single pion production}

Following the commissioning of CLAS our group developed a number of procedures and software for carrying out the initial CLAS data reduction, which have been detailed in previous reports. These include physics event generators, radiative corrections, simulation of CLAS acceptance and track reconstruction resolution, and kinematic cuts necessary for suppression of Bethe-Heitler backgrounds (in the case of $\pi^{0}$ electroproduction).

We have analyzed the $e p \rightarrow e^{\prime} p \pi^{o}$ reaction using data taken during the E1B and E1C run periods. We published a paper on the quadrupole $N \rightarrow \Delta$ transition for $Q^{2}<2.0 \mathrm{GeV}^{2}$ and a paper on single spin observables in the $\Delta(1232)$ region. We are also collaborating with Kyungseon Joo (U. of Conn.) on analysis of single spin observables using the $e p \rightarrow$ $e^{\prime} \pi^{+} n$ reaction in both the $\Delta(1232)$ and second resonance regions. The E1E run used beam energies of 1.045 and $2.037 \mathrm{GeV}$ to acquire new pion electroproduction data down to $Q^{2}=0.1 \mathrm{GeV}^{2}$ on both proton and deuterium targets. These data, which are currently undergoing calibration, should extend our current coverage in both $W$ and $Q^{2}$ for polarized beam asymmetry studies in the $\Delta(1232)$ and Roper resonance regions, and provide new information on the role of the pion cloud in the resonance excitation mechanism. 


\subsection{Experimental Results}

In the following sections we will discuss results obtained from the specific experiments listed below:

1. E89-037: "Electroproduction of the $\mathrm{P}_{33}$ resonance", (Burkert and Minehart).

2. E89-038: "Measurement of $\mathrm{p}\left(\mathrm{e}, \mathrm{e}^{\prime} \pi^{+}\right) \mathrm{n}, \mathrm{p}\left(\mathrm{e}, \mathrm{e}^{\prime} \mathrm{p}\right) \pi^{0}$, and $\mathrm{n}\left(\mathrm{e}, \mathrm{e}^{\prime} \pi^{-}\right) \mathrm{p}$ in the 2 nd and 3rd Resonance Regions", (Minehart, Burkert and Gai).

3. E89-042: "A measurement of the electron asymmetry in $\mathrm{p}\left(\vec{e}, \mathrm{e}^{\prime} \mathrm{p}\right) \pi^{0}$ and $\mathrm{p}\left(\vec{e}, \mathrm{e}^{\prime} \pi^{+}\right) \mathrm{n}$ in the mass region of the $\mathrm{P}_{33}(1232)$ ", (Burkert and Minehart).

The goal of E89-037 is to extract the small quadrupole component of the electromagnetic transition $\gamma^{*} N \rightarrow \Delta(1232)$, which has been interpreted as arising from a charge deformation in either the pion cloud or inner quark core of the $N / \Delta$ system. The goal of E89-038 is to study properties of higher mass states using the detailed shapes of the angular distributions and isotopic spin effects to disentangle individual resonant states and test the single quark transition model (SQTM) of resonance excitations. Experiment E89-042 makes use of a polarized electron beam, with the goal of extracting imaginary parts of multipole interference terms in the region of the $\Delta(1232)$. This experiment is complementary to the first one in the sense that these terms are primarily determined by the non-resonant part of the pion production. Their measurement is important in understanding the role of non-resonant mechanisms in modifying the resonance photocoupling amplitudes predicted by quark models.

The E1 run group completed data taking for the run periods: e1a,b,c,d and e1-6, during the winter of 2001-2002. At that time $75 \%$ of the approved data set for the experiments listed above had been taken, using beam energies over the range 1.5-6.0 GeV. First results from $\pi^{0}$ electroproduction of the $\Delta(1232)$ based on analysis by Kyungseon Joo and Cole Smith were published in Physical Review Letters in April 2002 [2]. Analysis of the $\pi^{+}$cross section data formed the basis for the Ph.D. thesis of Hovanes Egiyan [1] and those data have been released to analysis groups and a paper has been submitted for publication. Results for the polarized structure function $\sigma_{L T}^{\prime}$ in the $\Delta(1232)$ region for both $\pi^{0} p$ and $\pi^{+} n$ channels were published by Joo and Smith in Physical Review C in July 2003 [3] and Sept. 2004 [4], and a paper covering the second resonance region is in preparation [5]. A global analysis of both polarized and unpolarized data sets in the first and second resonance regions was published by Inna Aznauryan and our group in Jan. 2005 [6]. Higher energy $\pi^{0}$ and $\pi^{+}$ data sets covering broader ranges of $Q^{2}$ and $W$ were analyzed by Maurizio Ungaro [34] of RPI and Kijun Park of Kyungpook University. The analysis of the $\pi^{0}$ data have been published and the $\pi^{+}$analysis is awaiting final review.

\subsubsection{E89-037}

This experiment centers on measurement of the quadrupole strength in the $\gamma^{*} p \rightarrow \Delta(1232) \rightarrow$ $p \pi^{0}$ transition and its $Q^{2}$ dependence. This transition provides direct information on the shape of the hadron, which is predicted to be non-spherical in QCD inspired models and recent lattice calculations. A quadrupole deformation requires non-central forces within the hadron, which arise in QCD-motivated quark models through the tensor component of the 
one-gluon-exchange (OGE) potential between the constituent quarks (color magnetism). However the OGE mechanism generally predicts a smaller quadrupole deformation than measured experimentally.

At the same time, at sufficiently low $Q^{2}$ the dynamical breaking of chiral symmetry in QCD leads to the coupling of light Goldstone bosons to the quarks, which can be identified with the asymmetrically coupled charged $\pi$-meson cloud surrounding the nucleon. Chiral bag and soliton models, in which quark confinement occurs through non-linear interactions with the pion cloud, generally predict much larger quadrupole deformations compared to constituent quark models.

In 2002 we published [2] our results for the ratios $R_{E M}=E_{1+} / M_{1+}$ and $R_{S M}=$ $S_{1+} / M_{1+}$, of the electric and Coulomb quadrupole to the magnetic dipole, which were extracted using a partial wave analysis of the $p\left(e, e^{\prime} p\right) \pi^{o}$ reaction over a large range of fourmomentum transfer $Q^{2}=0.4-1.8 \mathrm{GeV}^{2}$. These results represented a substantial improvement in accuracy over previous measurements in this $Q^{2}$ interval.

Dynamical reaction models based on chiral effective field theories calculate the effects of pion re-scattering at the $\gamma N \Delta$ vertex. These models predict that meson degrees of freedom should strongly enhance the quadrupole strength at low $Q^{2}$. The trend of our results is consistent with the predictions from these models, although at low $Q^{2}$ the models begin to diverge, and more data are needed to constrain the calculations. In addition there are recent lattice QCD calculations of these ratios. Since the lattice calculations are quenched, they exclude the effect of sea quarks and pion cloud dynamics, although some of these effects may cancel in $R_{E M}$. Note the quark mass dependence of the lattice predictions has been chirally extrapolated to permit comparison to data. The most interesting comparison is for $R_{S M}$ for $Q^{2}<0.3 \mathrm{GeV}^{2}$, where the lattice prediction rapidly approaches zero, and is in disagreement with the Bates measurement at $Q^{2}=0.127 \mathrm{GeV}^{2}$. Our new data from the CLAS E1E run, discussed shortly, should provide new results in this region with continous coverage down to $Q^{2}=0.1 \mathrm{GeV}^{2}$.

In addition to the quadrupole deformation, mesonic dressing of the $\gamma^{*} p \rightarrow \Delta(1232)$ vertex also strongly affects the dominant magnetic dipole $M_{1+}$ photocoupling, and accounts for as much as $40 \%$ of the measured $M 1$ strength at low $Q^{2}$. The remaining $M_{1+}$ strength is attributed to a spin-flip transition of the constituent quarks. While the new CLAS data are approaching the precision necessary to distinguish between the dynamical model predictions of $M_{1+}$, the quadrupole ratios $R_{E M}$ and $R_{S M}$ still provide the greatest sensitivity to pionic effects. Interestingly, the quenched lattice calculation for $G_{M}^{*}$ correctly describes the $M_{1+}$ strength at the photon point $\left(Q^{2}=0\right)$, but predicts a somewhat 'harder' transition form factor, which would imply a smaller magnetic radius on the lattice compared to the physical $\Delta$.

Asymptotic pQCD power counting predicts that in the limit $Q^{2} \rightarrow \inf , R_{E M} \rightarrow 1$ due to dominance by the helicity conserving component of $M_{1+}$ and $E_{1+}$, and $R_{S M} \rightarrow$ constant. The CLAS data on $R_{E M}$ and $R_{S M}$ showed this limit is not yet reached at $Q^{2}=6 \mathrm{GeV}^{2}$. Corrections to the pQCD predictions can arise from the orbital angular momentum of quarks, which would destroy the correlation between quark and hadron helicity conservation. Idilbi, Ji and Ma [8] performed a leading order pQCD calculation of the helicity nonconserving amplitude which contributes to $S_{1+}$, claiming this quantity was computationally simpler than the double helicity flip amplitude contributing to $R_{E M}$. A correction of the form, $\log ^{2}\left(Q^{2} / \Lambda^{2}\right)$, arises from the orbital motion of small-x partons. However the CLAS 
results for $R_{S M}$ showed a substantially stronger $Q^{2}$ dependence, which may indicate a continued strong role of non-partonic or mesonic currents.

\subsubsection{E89-042}

This experiment used a polarized electron beam and the out-of-plane capability of CLAS to access the 'fifth' structure function $\sigma_{L T}^{\prime}$ in pion electroproduction, which measures the imaginary part of the interference of longitudinal and transverse amplitudes:

$$
\operatorname{Im}\left(L^{*} T\right)=\operatorname{Im}(L) \operatorname{Re}(T)-\operatorname{Re}(L) \operatorname{Im}(T)
$$

For kinematics where one of the two terms in Eq. 4 dominate, measurement of $\sigma_{L T}^{\prime}$ can serve to 'amplify' specific pion Born terms via interference with a strong resonance, or to increase sensitivity to weak resonances such as the Roper. The relative phase between different multipoles is also constrained, which is useful in fitting data above the $2 \pi$ threshold where Watson's Theorem can no longer be used to relate the real and imaginary parts of multipoles.

Our group published the first simultaneous measurements of this observable for both $\pi^{0}$ and $\pi^{+}$electroproduction. Fig. 4 shows an example of CLAS measurements [3, 4] of $\sigma_{L T}^{\prime}$ near the peak of the $\Delta(1232)$. This region is particularly interesting in view of the important role of meson rescattering in the excitation strength of the $\Delta(1232)$ discussed previously. The curves are dynamical model predictions which include non-resonant Born diagrams unitarized according to various prescriptions for handling off-shell $\pi N$ rescattering. The model predictions show smaller variation in the $\pi^{+} n$ channel, presumably because the rescattering corrections are smaller compared to the $\pi^{0} p$ channel. The forward peaking in the $\pi^{+}$channel is dominated by a direct interference of the $\Delta(1232)$ with the $t$-channel pion pole term. Interestingly, this pole term in also important for the $\pi^{0} p$ channel, which was verified by turning off this term in the Sato-Lee calculation.

\subsubsection{E89-038}

Measurements of $e p \rightarrow e^{\prime} \pi^{+} n$ are crucial for determining the photocoupling amplitudes for resonance transitions into isospin $I=1 / 2$ states, such as $P_{11}(1440), D_{13}(1520)$ and $S_{11}(1535)$, which dominate the second resonance region. Results from high precision CLAS $\pi^{+} n$ measurements [1] in the range $Q^{2}=0.3-0.7 \mathrm{GeV}^{2}$ are being prepared for publication. A new significant result using these data is discovery of a large sensitivity to the $M_{1-}$ and $S_{1-}$ multipoles (shown in Fig. 5), which contribute to excitation of the Roper $P_{11}(1440)$. Combining the $\sigma_{L T}^{\prime}\left(\pi^{+} n\right)$ data taken in the second resonance region with the cross section data, we have been able significantly constrain the real and imaginary parts of these multipoles through their interference, based on a unitary isobar model analysis [6], with the non-resonant Born pion pole term.

Through the unitary model fits [6] we have extracted the Roper photocoupling amplitudes at two $Q^{2}$ points, as shown in Fig. 6. These new data can completely rule out some previous quark model calculations, such as the quark-gluon hybrid approach of Li and Burkert which predicts that no zero crossing occurs in $A_{1 / 2}^{p}$ and that $S_{1 / 2}^{p}=0$. The strong longitudinal response seen in our data appears to revive the traditional 'breathing mode' interpretation of the Roper, but is also consistent with models in which a signficant mesonic 
component to the quark wave function is present. Another controversy centers around the possible role of the Roper as a member of a pentaquark anti-decuplet in the Jaffe-Wilcek diquark model. Precise measurements of the Roper form factor may help decide this question.

Similarly, combined analysis of unpolarized and polarized CLAS data above the Roper region have revealed strong longitudinal electromagnetic couplings for the $S_{11}(1535)$ and $D_{13}(1520)$ resonances (Fig. 7). Although pure quark models make non-zero predictions for longitudinal photcouplings of orbitally excited resonances, there is no consistent agreement with the data. Dynamical pion models have only begun to address the mesonic dressing of photocouplings in the second resonance region.

\subsubsection{Polarization observables}

Non-resonant backgrounds provide the largest source of uncertainty in the study of nucleon resonances. Sensitivity to backgrounds can be enhanced through the measurement of polarization observables which depend on beam helicity. In particular, the 'fifth' structure function $\sigma_{L T^{\prime}}$ probes the interference between real and imaginary amplitudes. Using a partial wave analysis, terms can be identified which represent the direct interference of resonant and non-resonant multipoles, and this amplification of weak backgrounds can be exploited to test model predictions.

In 2004 we completed our initial study of $\sigma_{L T^{\prime}}$ for both the $\pi^{0} p$ and $\pi^{+} n$ channels, in the invariant mass region around the $\Delta(1232)$ resonance. Results are shown in Figures 1 and 2. The center-of-mass (c.m.) angular distributions are compared with predictions from models which include the $\Delta(1232)$ resonance, plus Born diagrams, $t$-channel vector meson exchange and unitarized rescattering corrections. The $\pi^{+} n$ channel is dominated by the pion pole term, which is responsible for the forward peaking seen, while for the $\pi^{0} p$ channel this term is absent, although it can contribute via loop diagrams involving final or initial state rescattering. Clearly the model dependence is larger for the $\pi^{0} p$ channel. More data at lower $Q^{2}$ (Figure 2) are clearly needed to address the model dependence and should be forthcoming from the E1E run just completed.

The $\pi^{0} p$ analysis was published and the analysis of the $\pi^{+} n$ channel is awaiting final collaboration approval. In addition we extended the beam asymmetry analysis into the 2 nd resonance region in order to look for possible sensitivity to the P11 (Roper) resonance. First results using the beam asymmetry $A_{L T^{\prime}}$ were promising. In Figure 3 it can be seen that a better fit to the data in the region of the P11 can be achieved by adjusting the resonant $S_{1-}$ multipole, which is sensitive to the longitudinal coupling of the Roper.

\section{$1.4 N^{*}$ Low Energy Experiments}

The final 30 days of E1 running in Hall B included

1. 10 days to increase our statistics for single spin beam asymmetry data at low energies in order to study the $Q^{2}$ dependence of the Roper;

2. 10 days to extend our measurement of the $N \rightarrow \Delta(1232)$ quadrupole transition to very low $Q^{2}$ in order to study pion degrees of freedom more fully. 

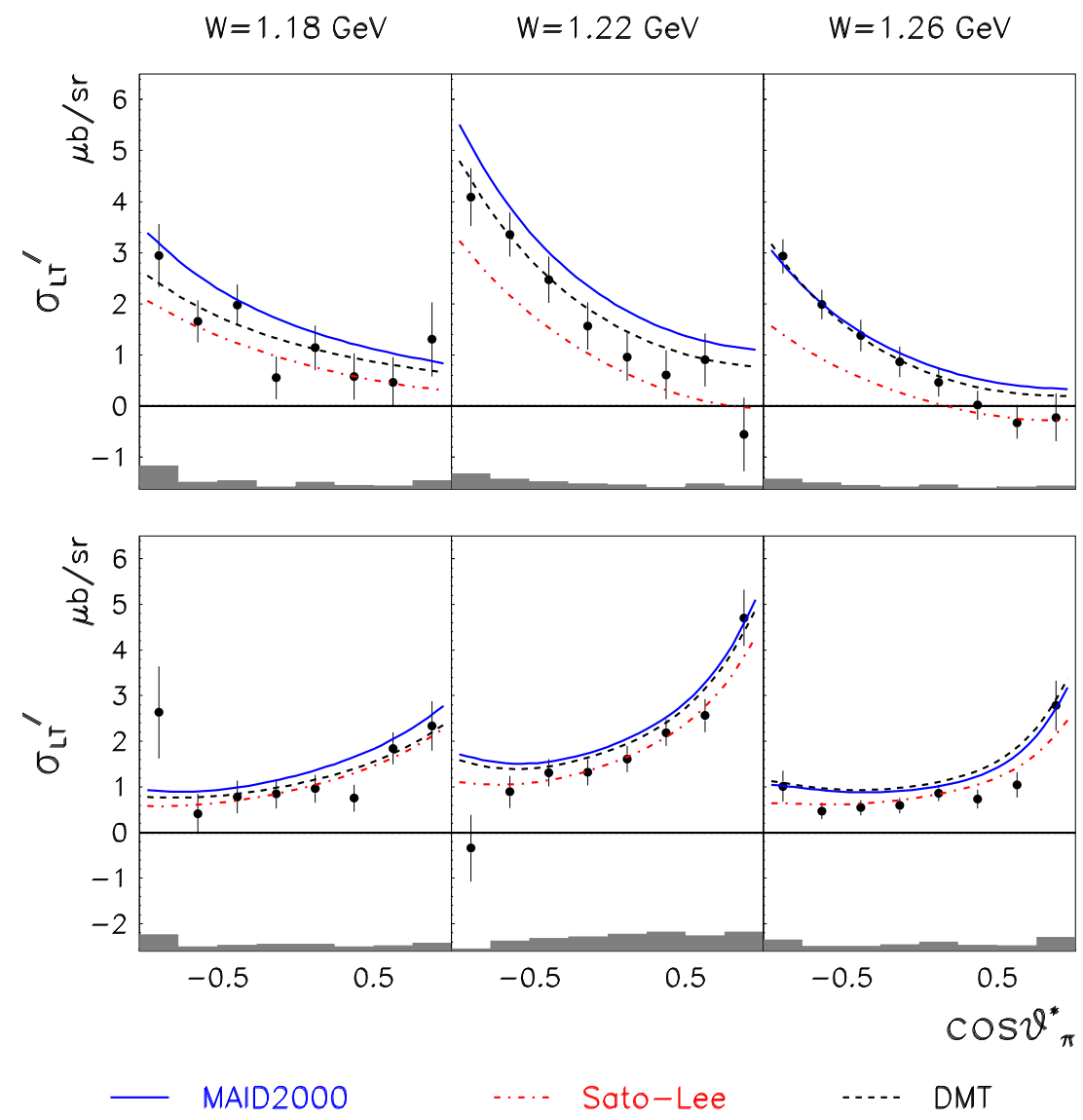

Figure 1: CLAS measurements of $\sigma_{L T^{\prime}}$ versus $\cos \theta_{\pi}^{*}$ for the $\pi^{0} p$ channel (top) the $\pi^{+} n$ channel (bottom) extracted at $Q^{2}=0.40 \mathrm{GeV}^{2}$. Curves show model predictions. Shaded bars show systematic errors

3. 10 days to complete measurements on liquid deuterium, which provides data from a quasi-free neutron target needed for a complete isospin analysis of resonance amplitudes.

These data were taken during the E1E run between Nov. 2002 and Jan. 2003, which used beam energies of $1.046 \mathrm{GeV}$ and $2.039 \mathrm{GeV}$..

The analysis was shared by C. Smith at UVa and K. Joo at the University of Connecticut. The UVa program concentrated low $Q^{2}$ analysis of both $\pi^{0}$ and $\pi^{+}$channels in the $\Delta(1232)$ region, for both $\mathrm{H}_{2}$ and $\mathrm{D}_{2}$ targets. Analysis of the deuteron data served several purposes. For detector calibration it provides a means of calibrating the proton detection efficiency of CLAS through the $d\left(e, e^{\prime} p \pi^{-}\right) p$ reaction, by tagging the missing proton through detection of the proton and $\pi^{-}$. Second, the data can be used to test Single Quark Transition Model (SQTM) predictions of the isospin structure of the $N^{*}$ transition currents. Single pion electroproduction proceeds through both isoscalar $A^{(0)}$ and isovector $A^{(3 / 2)}, A^{(1 / 2)}$ amplitudes and information about the isoscalar photon excitation can only be achieved by 


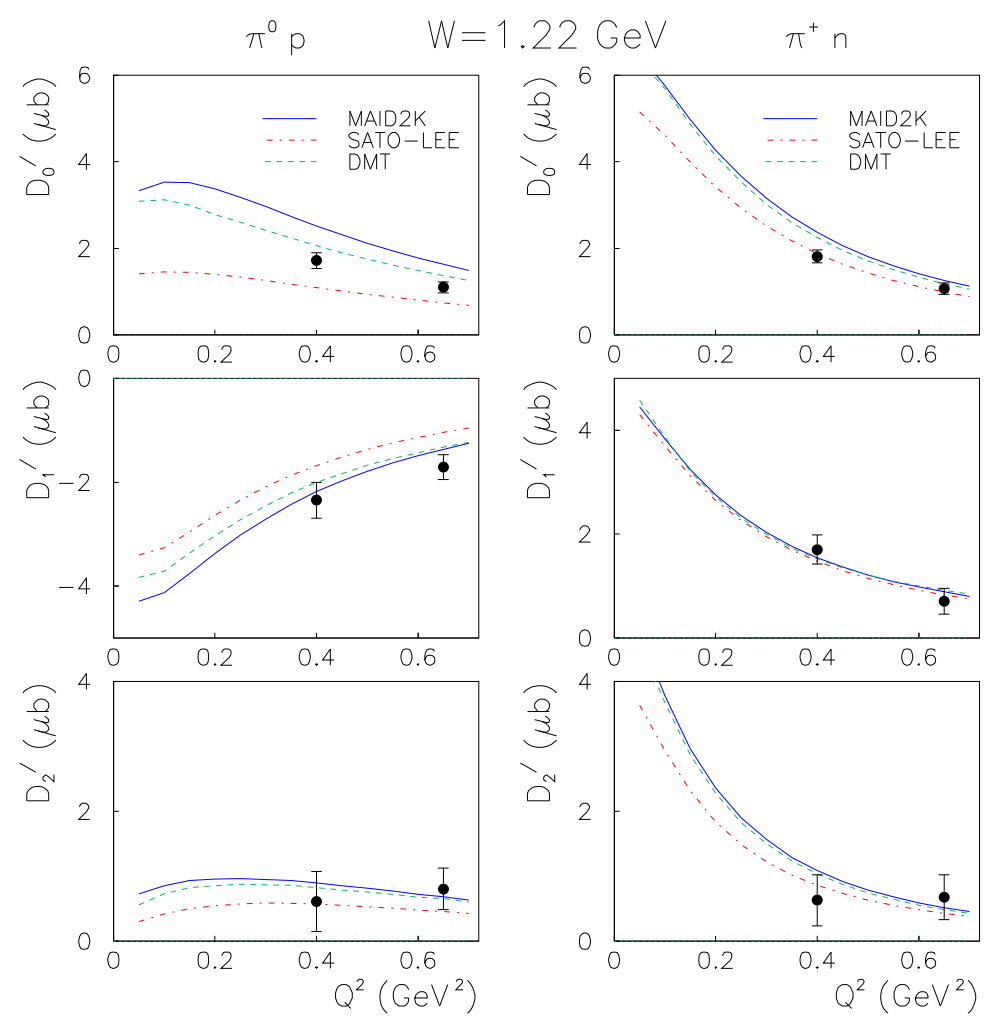

Figure 2: $Q^{2}$ dependence of Legendre moments of $\sigma_{L T^{\prime}}$ for $\pi^{0} p$ channel (left) and $\pi^{+} n$ channel (right). Curves show model predictions. Data points are the present CLAS measurement. Statistical error only.

combining measurements from both a proton and neutron target:

$$
\begin{aligned}
& A\left(\gamma^{*} p \rightarrow n \pi^{+}\right)=\sqrt{2}\left[A^{(0)}+\frac{1}{3} A^{(1 / 2)}-\frac{1}{3} A^{(3 / 2)}\right] \\
& A\left(\gamma^{*} n \rightarrow p \pi^{-}\right)=\sqrt{2}\left[A^{(0)}-\frac{1}{3} A^{(1 / 2)}+\frac{1}{3} A^{(3 / 2)}\right]
\end{aligned}
$$

For the $\Delta(1232)$ resonance, this information can help reduce model dependence in separating the resonant $I=3 / 2$ parts of the $E_{1+}$ and $S_{1+}$ quadrupole transitions from the Born dominated backgrounds. For higher energy excitations, precision measurements of both neutron and proton target multipoles can test SQTM predictions of a non-zero spin-orbit term in the transition current of members of the $\left[\mathbf{7 0}, \mathbf{1}^{-}\right]$multiplet. For example, $A_{p}^{(3 / 2)}=$ $-A_{n}^{(3 / 2)}$ for the $D_{13}(1520)$ in the absence of spin-orbit effects. While the data appear to favor the spin-orbit term, little information is available on the isoscalar/isovector decomposition and its $Q^{2}$ dependence.

The data at 1.046 and $2.039 \mathrm{GeV}$ will complement earlier measurements at $2.4 \mathrm{GeV}$ and above $4 \mathrm{GeV}$ which will be analyzed as a single group. 


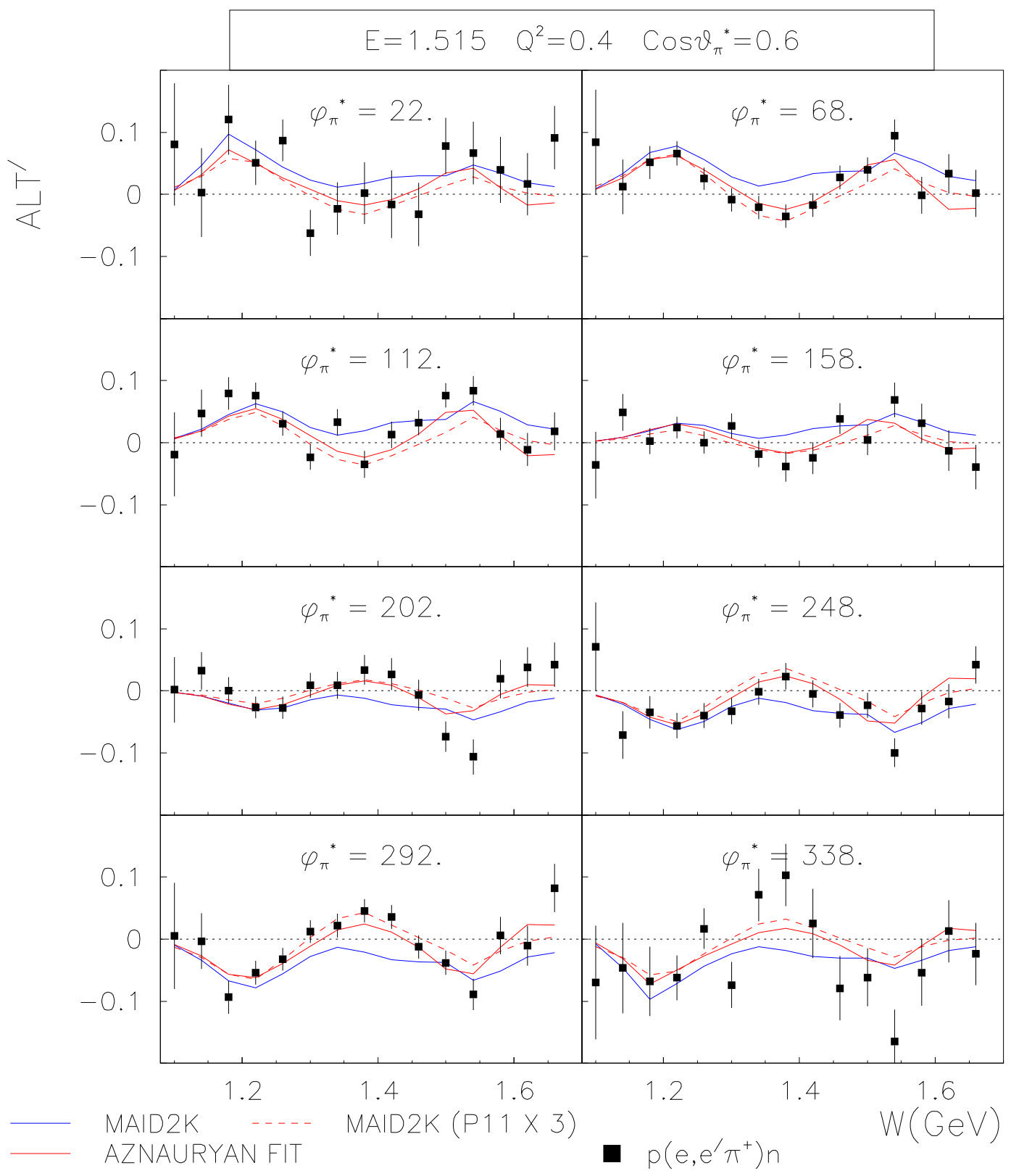

Figure 3: Beam asymmetry $A_{L T^{\prime}}$ for the $e p \rightarrow e^{\prime} \pi^{+} n$ reaction. The $W$ dependence is plotted for various bins in $\phi_{\pi}^{*}$. Blue curve show the prediction of MAID with the default Roper strength and with the Roper strength increased by a factor of three (dashed curve) in the $S_{1-}$ longitudinal multipole. Red curve shows the fit of Aznauryan, which also requires a non-zero longitudinal Roper strength. 

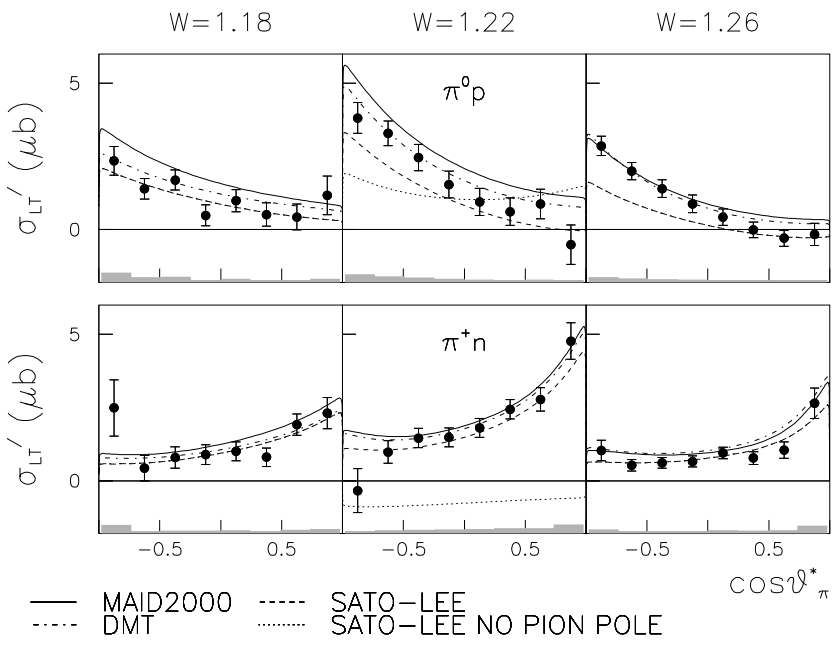

Figure 4: CLAS measurements of $\sigma_{L T^{\prime}}$ versus $\cos \theta_{\pi}^{*}$ for the $\pi^{0} p$ channel [3] (top) and for the $\pi^{+} n$ channel [4](bottom) extracted at $Q^{2}=0.40 \mathrm{GeV}^{2}$ and $W=1.18-1.26 \mathrm{GeV}$. The curves show model predictions. The shaded bars show estimated systematic errors. 


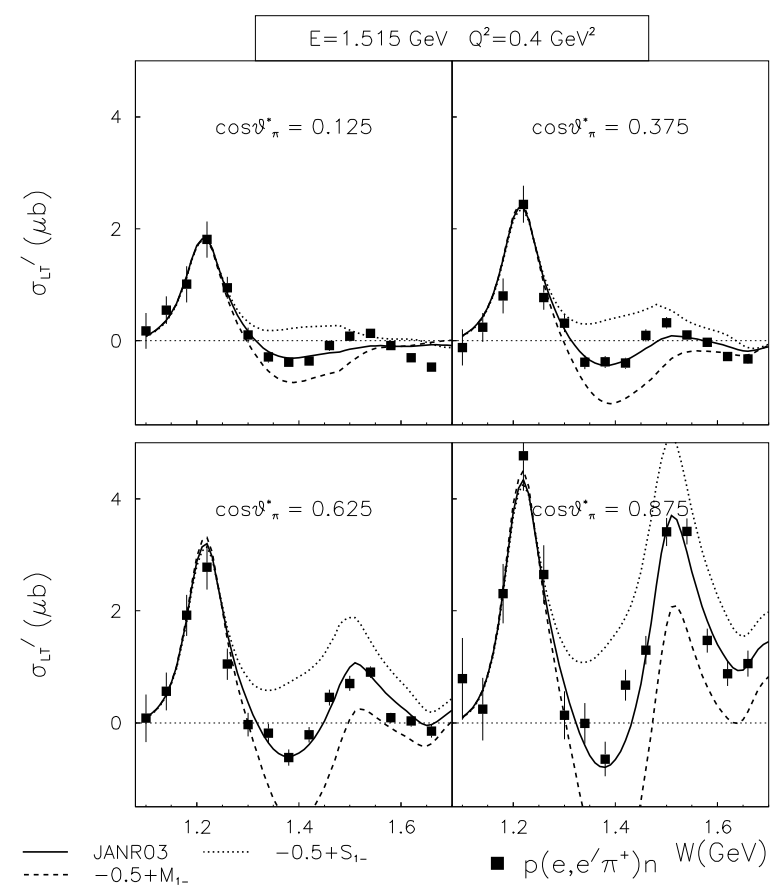

Figure 5: Sensitivity of $\sigma_{L T}^{\prime}\left(\pi^{+} n\right)$ to changes in Roper multipoles $M_{1-}$ and $S_{1-}$ using unitary isobar model of Aznauryan [6]. Solid line shows best fit.
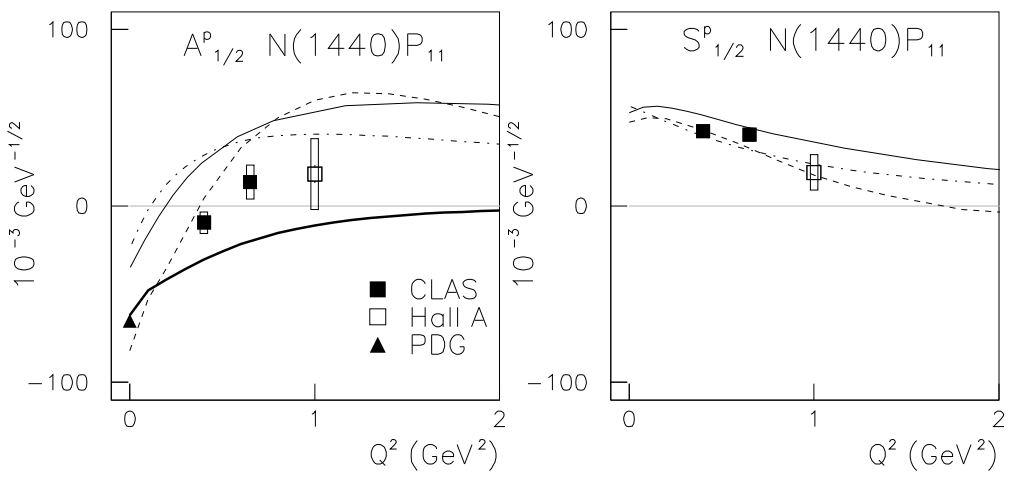

Figure 6: $Q^{2}$ dependence of transverse $A_{1 / 2}^{p}$ and longitudinal $S_{1 / 2}^{p}$ helicity amplitudes for the Roper $P_{11}(1440)$. Curves show recent quark model calculations. 

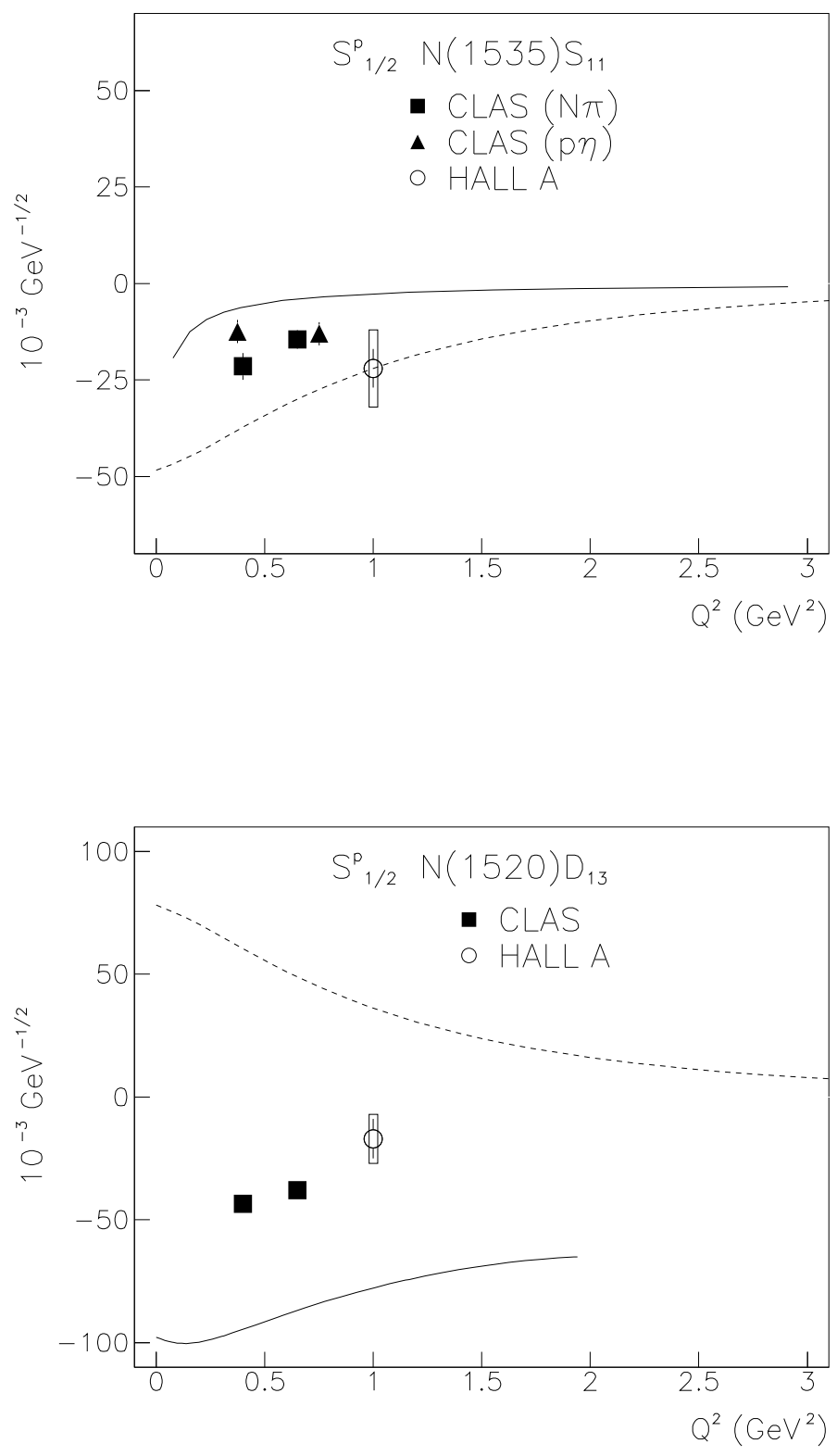

Figure 7: $Q^{2}$ dependence of longitudinal $S_{1 / 2}^{p}$ helicity amplitudes for the $S_{11}(1535)$ and $D_{13}(1520)$ resonances from global analysis of CLAS $\pi^{+}$and $\pi^{0}$ electroproduction data. Curves show recent quark model calculations. 

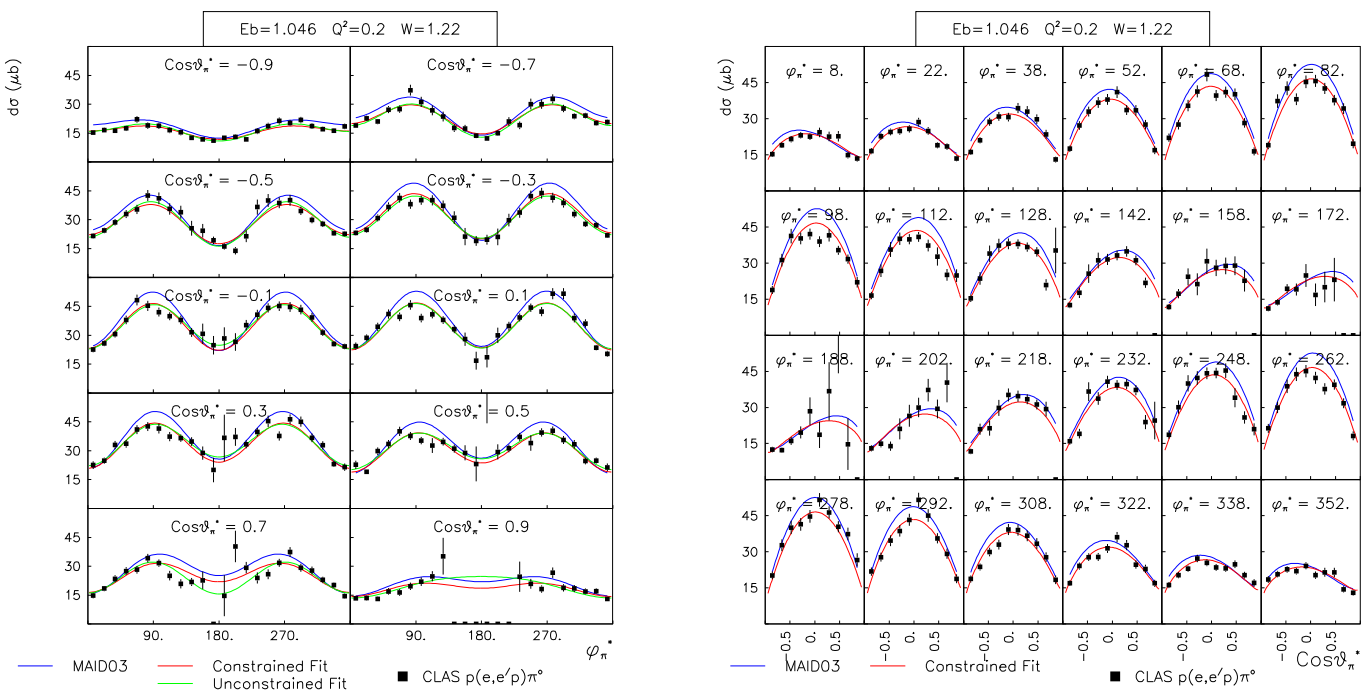

Figure 8: Preliminary measurements of $p\left(e, e^{\prime} p\right) \pi^{0}$ at $Q^{2}=0.2 \mathrm{GeV}^{2}$ from 2002 CLAS E1E run. Plot at left shows angle $\phi_{\pi^{0}}^{*}$ of $p \pi^{0}$ hadronic decay plane relative to $\left(e, e^{\prime}\right)$ plane. Plot at right shows $p \pi^{0}$ center-of-mass angle $\cos \left(\theta_{\pi^{0}}^{*}\right)$.

\section{$2 \quad$ EG1a and EG1b}

\subsection{The Physics of Double Polarization Experiments}

The study of the spin structure of the nucleon through inclusive electron scattering has been a major subject of interest ever since the discovery from deep inelastic scattering (DIS) measurements that only about $25 \%$ of the nucleon spin could be attributed to the intrinsic spin of the quarks [9]. Inclusive spin dependent electron scattering can be described in terms of two spin structure functions, $g_{1}$ and $g_{2}$, which at asymptotic momentum and energy (the scaling region) each depend (except for small corrections) on the Bjorken scaling variable, $x=Q^{2} / 2 M$. The Bjorken sum rule [10] relates the proton-neutron difference of the first moment of the spin structure function $g_{1}, \Gamma_{1}$, defined as:

$$
\Gamma_{1}=\int g_{1}(x) d x
$$

to the weak axial coupling constant $g_{A}$ :

$$
\Gamma_{1}^{p}-\Gamma_{1}^{n}=\frac{1}{6} g_{A}
$$

where the superscripts, $p$ and $n$, denote proton and neutron, respectively. The Bjorken sum rule has been evolved in perturbative QCD to finite $\mathrm{Q}^{2}$ and has been verified experimentally at the $5 \%$ level. However, the fact that only a small fraction of the nucleon spin can be directly attributed to the quark spin is in contradiction to the expectations of the quark model of the nucleon, and shows that we are far from understanding the structure of the nucleon. Although the experiments are difficult, the exploration of the spin structure in the 
resonance regime at low $\mathrm{Q}^{2}$ is essential to a complete understanding of nucleon structure. This is the domain where JLab experiments will have their greatest impact.

The differential cross section for scattering of longitudinally polarized electrons from longitudinally polarized protons can be written as:

$$
\frac{d \sigma}{d \Omega_{e} d E_{e}^{\prime}}=\Gamma_{T}\left[\sigma_{T}+\epsilon \sigma_{L}+P_{e} P_{t} \sigma_{e t}\right]
$$

where $P_{e}$ and $P_{t}$ are the electron and proton polarizations, $\Gamma_{T}$ is the transverse flux factor, $\epsilon$ is the virtual photon polarization, and $\sigma_{T}\left(W, Q^{2}\right)$ and $\sigma_{L}\left(W, Q^{2}\right)$ are the absorption cross sections for transverse and longitudinal virtual photons. The double polarization absorption cross section is given by:

$$
\sigma_{e t}=\sqrt{1-\epsilon^{2}} \cos \theta_{\gamma} A_{1} \sigma_{T}+\sqrt{2 \epsilon(1-\epsilon)} \sin \theta_{\gamma} A_{2} \sigma_{T},
$$

where $\theta_{\gamma}$ is the angle between the virtual photon and proton, $A_{1}\left(Q^{2}, W\right)$ is the transverse asymmetry for the helicity $\frac{1}{2}$ and $\frac{3}{2}$ states of the virtual photon and proton, and $A_{2}\left(Q^{2}, W\right)$ arises from transverse-longitudinal interference. Writing $\sigma_{T}=(1 / 2)\left(\sigma_{T}^{1 / 2}+\sigma_{T}^{3 / 2}\right)$ where the superscript denotes scattering in the helicity $1 / 2$ and $3 / 2$ states of the virtual photon and proton:

$$
A_{1}\left(Q^{2}, W\right)=\frac{\sigma_{T}^{1 / 2}-\sigma_{T}^{3 / 2}}{2 \sigma_{T}}, \quad A_{2}\left(Q^{2}, W\right)=\frac{\sigma_{L T}}{\sigma_{T}} .
$$

In the resonance region, the relative contributions of $\sigma_{T}^{1 / 2}$ and $\sigma_{T}^{3 / 2}$ vary from one resonance to another and depend strongly on $\mathrm{Q}^{2}$.

$\mathrm{A}_{1}$ and $\mathrm{A}_{2}$ are linearly related to the spin structure functions, $g_{1}$ and $g_{2}$, with $g_{1}$ given explicitly by:

$$
g_{1}\left(x, Q^{2}\right)=\frac{\tau}{1+\tau}\left(A_{1}+\frac{1}{\sqrt{\tau}} A_{2}\right) F_{1}\left(x, Q^{2}\right),
$$

where $\tau \equiv \frac{\nu^{2}}{Q^{2}}, x=\frac{Q^{2}}{2 M \nu}$ is the Bjorken scaling variable, and $F_{1}$ is a structure function known from unpolarized electron scattering measurements.

At $\mathrm{Q}^{2}=0$, the Gerasimov-Drell-Hearn $(\mathrm{GDH})$ sum rule [11] relates the differences in the helicity-dependent photo-absorption cross sections to the anomalous magnetic moment $\kappa^{2}$ of the target:

$$
\int_{\nu_{0}}^{\infty} \frac{\sigma_{1 / 2}(\nu)-\sigma_{3 / 2}(\nu)}{\nu} d \nu=-\frac{2 \pi^{2} \alpha \kappa^{2}}{M^{2}},
$$

where $\nu_{0}$ is the photon energy at pion threshold and $M$ is the target mass. The sum rule has been studied for photon energies up to $850 \mathrm{MeV}$ [12], and is currently being tested up to more than $2 \mathrm{GeV}$ at ELSA [13]. A rigorous extension of the GDH sum rule to non-zero $\mathrm{Q}^{2}$ as been carried out using chiral perturbation theory by Ji and Osborne [14] and Bernard et al. [15].

At high $Q^{2}, \Gamma_{1}$ is known from DIS experiments to be positive. At $\mathrm{Q}^{2}=0$, if the contribution from elastic scattering $(x=1)$ is excluded, $\Gamma_{1}$ vanishes, and the GDH sum rule sets its slope with $\mathrm{Q}^{2}$ to be negative. Therefore, $\Gamma_{1}$ must change sign at some low $\mathrm{Q}^{2}$, where it is dominated by transitions to the nucleon resonances.

The measurements of the helicity dependence of the inclusive electron scattering will provide stringent tests of nucleon structure models. It is also possible that comparison 
of low $\mathrm{Q}^{2}$ cross sections in the resonance region will shed new light on the well-known phenomenon of "duality" between the deep inelastic regime and the resonance regime that has been observed for the unpolarized structure function $F_{1}\left(x, Q^{2}\right)$. If duality is valid, the low $Q^{2}$ measurements can be used to study the behavior of $A_{1}$ for which there are distinctly different model predictions at high $\mathrm{Q}^{2}$ as $x \rightarrow 1$. In any event, a measurement of the spin-dependent cross sections for low and moderate $\mathrm{Q}^{2}$ will determine the distance scale at which pQCD corrections and higher twist expansions break down, and show where the contribution of resonances are important or even dominant [16] [17]. The rapidly changing helicity structure [18] of some resonances with the distance probed should be observable.

\subsection{CLAS Experiments}

Scattering of longitudinally polarized electrons from longitudinally polarized protons and deuterons, using dynamically pumped polarized $\mathrm{NH}_{3}$ and $\mathrm{ND}_{3}$ targets in the CLAS, was measured in two runs, the first (EG1a) in the fall of 1998, and the second (EG1b) from Sept. 2000 to April 2001. Improvements in the target increased the polarizations reached in the second run, for which about eight times more data were collected.

To extract spin dependent cross sections terms from the data, the straightforward approach is to measure cross sections for each sign of $P_{e}$ and $P_{t}$ and take appropriate differences to isolate the various terms. The $5 \mathrm{~T}$ magnetic polarizing field in the magnet and the uncertainties in the detector efficiencies makes precise determination of the acceptances of the apparatus difficult. An alternative method that is commonly chosen is to define an experimental asymmetry:

$$
A_{e x p}=\frac{N^{\downarrow \Uparrow}-N^{\uparrow \Uparrow}}{N^{\downarrow \Uparrow}+N^{\uparrow \Uparrow}-N_{b k g}},
$$

The single arrow refers to the helicity of the incident electrons, and the double arrow to the direction of polarization of the protons. The terms, $N^{\downarrow \Uparrow}$ and $N^{\uparrow \Uparrow}$, are counts (normalized to equal beam flux) measured in a given $\left(Q^{2}, W\right)$ kinematical bin for the electron and proton spins anti-parallel and parallel, respectively. The acceptance and efficiencies of each kinematical bin cancel out in the ratio.

In our experiments the target polarization was kept fixed over a long period of time, while the electron polarization alternated at $30 \mathrm{~Hz}$. Separate calculation of the asymmetry for each orientation of the target polarization provides an excellent check on systematic errors.

The major drawback of the asymmetry method is that while the numerator is determined almost entirely by scattering from the polarized free protons or deuterons in the target, the background counts, $N_{b k g}$, due to scattering from unpolarized ${ }^{15} \mathrm{~N},{ }^{4} \mathrm{He}$, and windows, makes a large contribution to the denominator. Typically:

$$
N_{b k g} \approx .88\left(N^{\downarrow \Uparrow}+N^{\uparrow \Uparrow}\right) .
$$

This background was measured by taking data with a carbon target and an empty (volume filled with helium) target and using a model to calculate the contribution of ${ }^{15} \mathrm{~N}$.

The photon absorption functions $A_{1}$ and $A_{2}$ are connected to the experimental asymmetry through the relation:

$$
A_{\text {exp }}=P_{e} P_{t} \sqrt{1-\epsilon^{2}} \cos \theta_{\gamma}\left[\frac{A_{1}+\eta A_{2}}{1+\epsilon \sigma_{L} / \sigma_{T}}\right],
$$


where $\eta$ is a kinematical quantity.

The CLAS detector provides excellent identification of the scattered electron and an accurate measurement of its energy and scattering angle. Small correctiong to the momentum can be obtained from a study of elastically scattered electrons, for which there is a strict correlation between the scattering angle and the electron momentum as well as between the scattering angle and the recoil proton kinematics. A fiducial region in the electron scattering phase space $(\theta, \phi)$ was defined to eliminate effects of particle interactions in the CLAS magnet coils and support structures.

The scattered electrons were binned in $\mathrm{W}$ and $\mathrm{Q}^{2}$, the counts were normalized according to the beam flux measured with a Faraday cup. Measurements with a thin carbon target and with no target are used to estimate the contribution of the nitrogen, the target cell walls, and the helium in the target to the term $N_{b k g}$. The beam polarization $P_{e}$ was periodically measured with a Moller polarimeter. The product of the beam and target polarization $P_{e} P_{t}$ was obtained by comparing the measured elastic electron scattering asymmetry to a parametrization of the world data.

Experimental asymmetries, defined in eqn. 15, are used to obtain the combination $A_{1}+\eta A_{2}$ for the proton. The measured asymmetries are corrected for radiative effects. The two methods used in analyzing the data, the asymmetry method and the absolute cross section difference method, yielded statistically consistent results. Because of its higher statistical accuracy only the results of the asymmetry method have been published. 


\subsection{Determination of Inclusive Structure Functions}

Using a parametrization of world data on ep scattering to determine $F_{1}$ and a combination of data and a model to calculate $A_{2}$ and $R$, the dominant $A_{1}$ (and $g_{1}$ ) can be extracted from our measurement of $A_{1}+\eta A_{2}$. The model makes use of existing data along with reasonable assumptions about the $Q^{2}$ and $x$ dependence of the structure functions. Results from EG1a for $g_{1}^{p}$ for $Q^{2}=1 \mathrm{GeV}^{2}$ bin are shown in Fig. 9. The values of $g_{1}^{p}$ using our model is shown as a solid line.

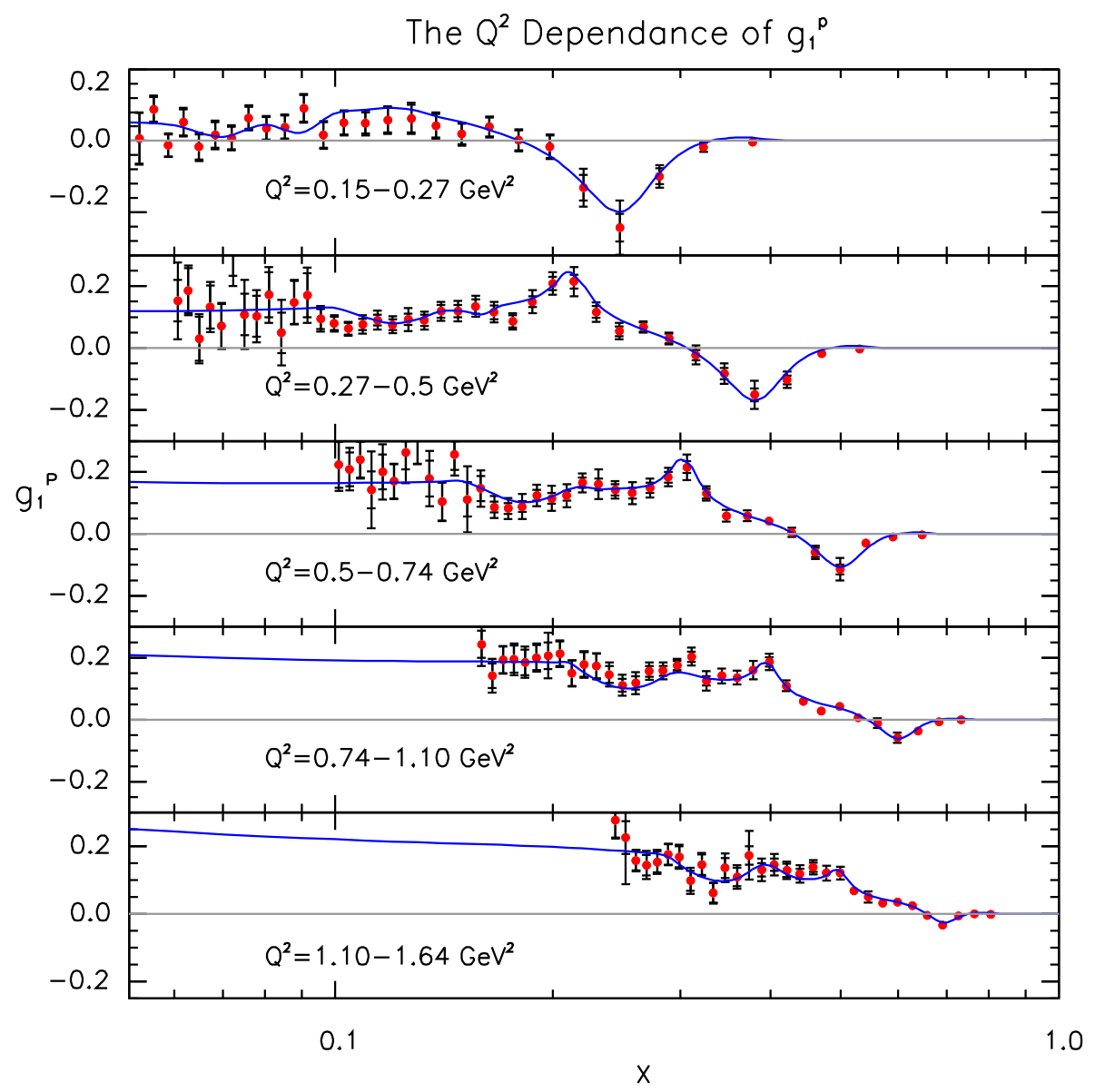

Figure 9: Eg1a measurements of $g_{1}(x)$ vs. $\mathrm{x}$ for the proton, for five $\mathrm{Q}^{2}$ bins. The solid line is a calculation using the parametrization described in the text. Double bars indicate statistical and (statistical + systematic) errors.

We can integrate the $g_{1}\left(x, Q^{2}\right)$ obtained in this way to obtain the contribution of our data to the integral $\Gamma_{1}$ (the contribution of elastic scattering is excluded). The result obtained by integrating up to $\mathrm{W}=2 \mathrm{GeV}$ is shown in Fig. 10(a) where it is compared to two calculations. The AO program[20] developed at JLab and the MAID program[21] developed at Mainz are phenomenological models based on parametrizations of existing unpolarized electro- and 
photo-production data. They can be used to predict the polarization observables as well as the unpolarized cross sections. The MAID model calculation shown in the figure includes only the resonance contribution to single meson production, while the AO calculation includes all resonance decay modes. The unmeasured low $x(W>2 \mathrm{GeV})$ contributions to the integral can be estimated by using the previously mentioned parametrization of world data. The combination of our data and the unmeasured contribution is shown in Figure 10(b). In this figure a number of predictions for the full integral are indicated. The negative slope at $\mathrm{Q}^{2}=0$ required by the GDH sum rule is indicated as is the low $\mathrm{Q}^{2}$ behavior predicted by the chiral perturbation calculation of Ji, Kao and Osborne[22], and by Bernard, Hemmert and Meissner[15]. The evolution of the deep inelastic scattering results to low $\mathrm{Q}^{2}$ obtained from higher twist calculations of pQCD [24] is shown as a line at high $Q^{2}$. The calculations of Soffer and Teryaev[23] (upper dashed line) and Burkert and Ioffe[17] (lower dashed line) in the intermediate region are also shown. The expected reversal in sign of $\Gamma_{1}$ occurs near $Q^{2}=0.25 \mathrm{GeV}^{2}$. Our results, however, for $\Gamma_{1}\left(\mathrm{Q}^{2}\right)$ are clearly outside the predictions from the pQCD evolution from DIS. They are in better agreement with the model calculations of Burkert and Ioffe that include s-channel baryon resonance excitations explicitly.

Systematic uncertainties were studied in detail. These included the uncertainty in the method used to remove the nuclear contribution from the $\mathrm{NH}_{3}$ spectra, the effects of cuts used to select a clean electron sample, the effect of target vertex cuts, etc. The contribution of model uncertainties in the estimation of $F_{1}, R$ and $A_{2}$ have been studied by using a number of alternate parameterizations, as well as by setting $R$ and $A_{2}$ to extreme values.

The analysis of data at incident beam energies of 1.6 and $5.7 \mathrm{GeV}$ from EG1b has been finished. The quality and range of the data are illustrated by plots of $g_{1}$ for the proton in Figs. 11-14. At the lower $Q^{2}$ the negative values associated with the excitation of the $\Delta(1232)$ are prominent. At the higher $Q^{2}$ the effect of the $\Delta$ is nearly gone, and the data agree well with the results of Deep Inelastic Scattering, indicating that duality sets in around $Q^{2}=1$. The EG1b results for $\Gamma_{1}$ are shown in Figs. 15 and 16. These data are consistent with EG1a, but more precise. Our measurements extend down to $Q^{2}=0.05$, providing an excellent picture of the behaviour of $\Gamma_{1}$ at very low $Q^{2}$.

Our data provide a good measurement of the transition from the low $Q^{2}$ region dominated by coherent transitions to the nucleon resonances to the deep inelastic region dominated by photon absorption on single quarks. In the deep inelastic region the ratio of $g_{1} / F_{1}$ is nearly constant as a function of $Q^{2}$. This behavior was observed in the SLAC E155 experiment. We can plot the same ratio for our data as a function of $Q^{2}$, as shown in Figs. 17 and 18 for various values of Bjorken $x$. Except at the highest $x$, our measured ratios agree with the DIS values for $Q^{2}>\sim 1 \mathrm{GeV}^{2}$. The constant ratio extends to lower $Q^{2}$ as $x$ decreases. Squares on the proton plot and arrows on the plots indicate the value of $Q^{2}$ for each value of $x$ at which the invariant mass $W=2 \mathrm{GeV}$. Ratios at smaller $x$ correspond to lower values of $W$. The position of the $\Delta(1232)$ is shown on the proton plot as a triangle. At this value of the invariant mass, the deviation from the DIS ratio is extreme, with the ratio being close to 0 . In general it can be seen from these plots the the breakdown in the constant ratio observed in DIS occurs for $W<2 \mathrm{GeV}$, which is the resonance region.

It has been observed in studies of the inclusive differential cross section in the resonance, that when an average curve is drawn through the cross section peaks associated with resonance production, the structure function $F_{1}(x)$ in the resonance region at relatively low $Q^{2}$ is well represented by the $F_{1}(x)$ measured in DIS. This phenomenon is known as 

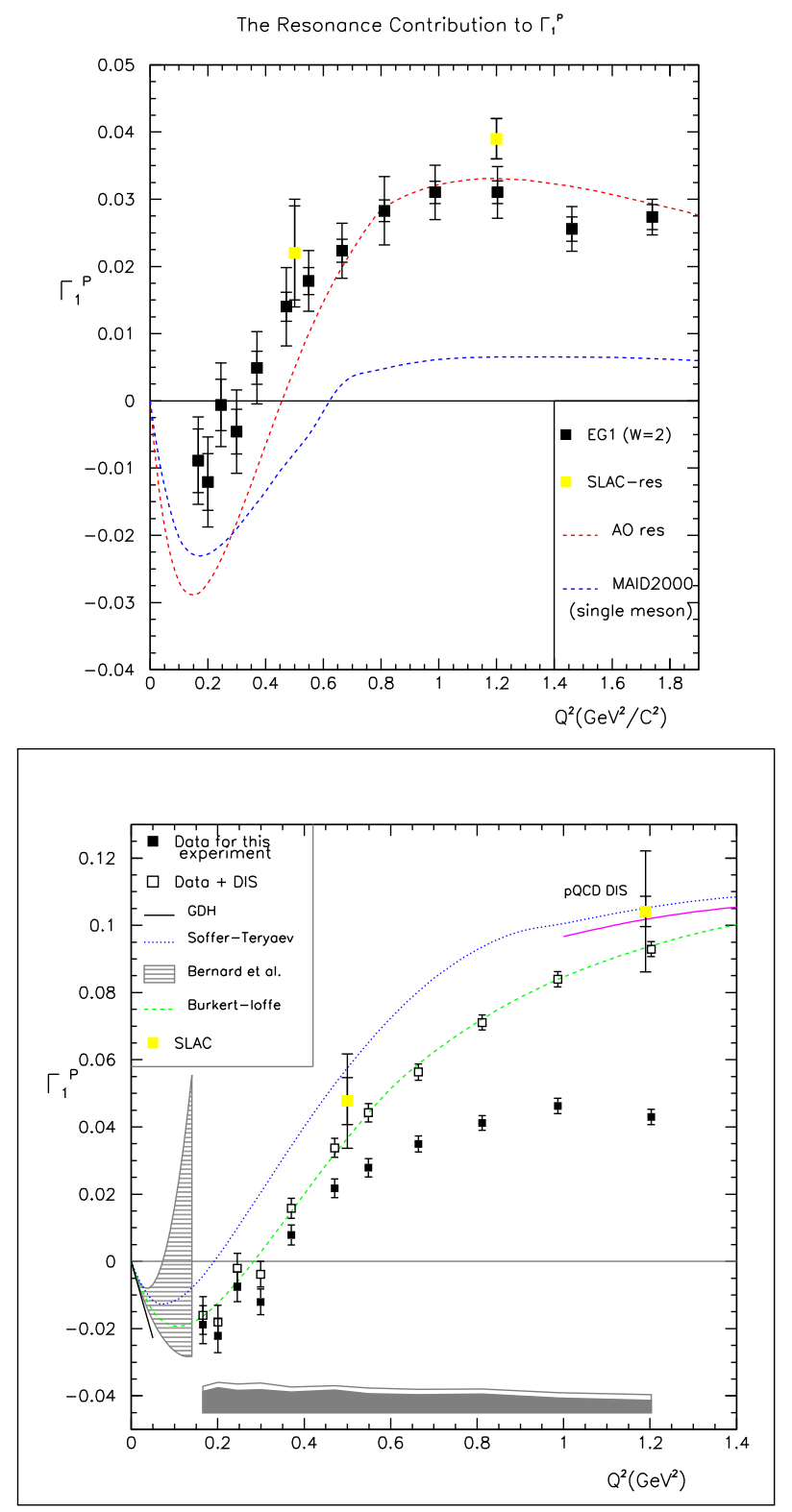

Figure 10: Eg1a measurements of $\Gamma_{1}$ vs. $Q^{2}$ for the proton. Double bars indicate statistical and (statistical + systematic) errors. (a) Solid circles show the integration up to $\mathrm{W}=2 \mathrm{GeV}$, using only our measurements. Data from SLAC are shown with open circles. The upper dashed line is a calculation using the AO program incorporating resonance production with $\mathrm{W}<2.0 \mathrm{GeV}$. The lower dashed line is a calculation using the MAID model for single meson production only. (b) The inclusive scattering model is used to extend the integral to very low $x$. Solid points are the same as in (a), the open circles include the integration to low $x$. Also shown are two points from SLAC $\left(\mathrm{Q}^{2}<=1.5\right)$. See the text for an explanation of the curves. 
Bloom-Gilman Duality, and is not completely understood. The agreement is even better if the structure functions are plotted as a function of the Nachtmann variable, $\xi$, which takes into account target recoil effects and agrees with $x$ at high $Q^{2}$. We can ask whether the spin structure functions obey a similar scaling. We can answer part ot the question immediately by noting that $g_{1}$ for transition to the $\Delta(1232)$ is negative whereas $g_{1}$ in DIS is everywhere positive. Scaling cannot work in the region of the $\Delta(1232)$ unless $g_{1}$ is neglible there. Our measurements of $g_{1}\left(\xi\right.$ are compared to the DIS function for various $Q^{2}$ bins in Fig. 19. At low $Q^{2}$ the negative values associated with the $\Delta(1232)$ are prominent. The excitation strength of the $\Delta(1232)$ dwindles with increasing $Q^{2}$, so that for $Q^{2}>1$ the DIS values fit our measurements very well.

Encouraged by these observations we have used our measurements in the invariant mass region of $W>2 \mathrm{GeV}$ to calculate the photon asymmetries, $A_{1}^{p}\left(x, Q^{2}\right)$, for $0.1<x<0.7$, and $A_{1}^{d}\left(x, Q^{2}\right)$. These results are plotted along with data from other experiments in Figs. 20 and 21. These plots also show the predictions of several models, along with the value at $x=1$ predicted by SU(6) symmetry and the value predicted by perturbative QCD (pQCD). The trends of the data appear to favor the pQCD limit, and are in fairly good agreement with the hyper-fine perturbed quark model (HFQM) of Isgur et al., [25].

We have published the inclusive scattering results of EG1a for the proton in Phys. Rev. Lett. [26] and for the deuteron data in Phys. Rev. C. [27]. A CLAS analysis paper for EG1b $(1.6$ and $5.7 \mathrm{GeV})$ is complete, and a PRL paper is under review by the CLAS collaboration. 


\section{$g_{1}^{p}$ with $\mathrm{E}=1.6 \mathrm{GeV}$ (preliminary)}

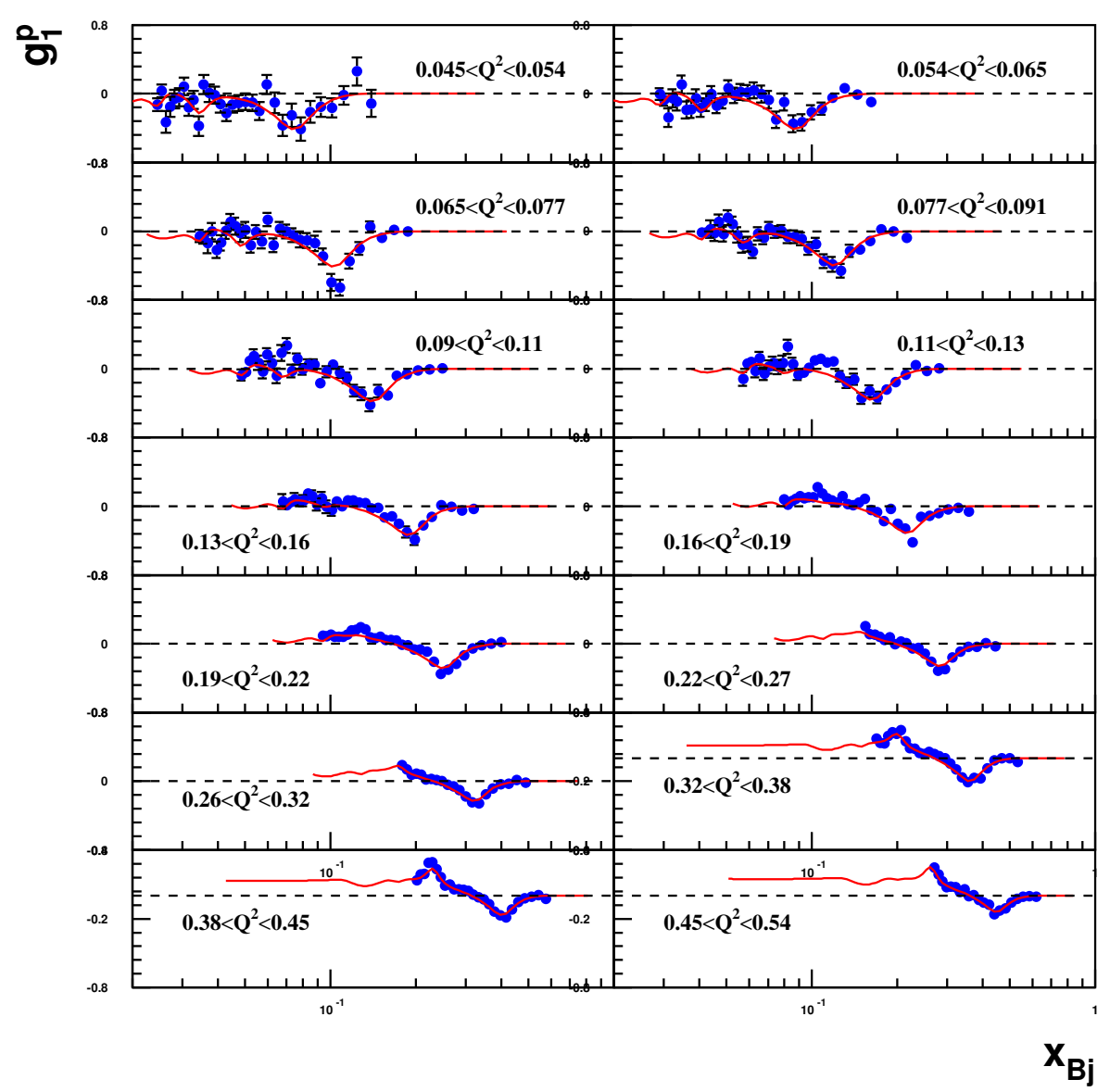

Figure 11: $g_{1}(x)$ vs. $\mathrm{x}$ for the proton, for $\mathrm{E}=1.6 \mathrm{GeV}$ (EG1b). The solid line is a calculation using the parametrization described in the text. Errors are statistical only. 


\section{$g_{1}^{p}$ with $\mathrm{E}=5.6 \mathrm{GeV}$ (preliminary)}

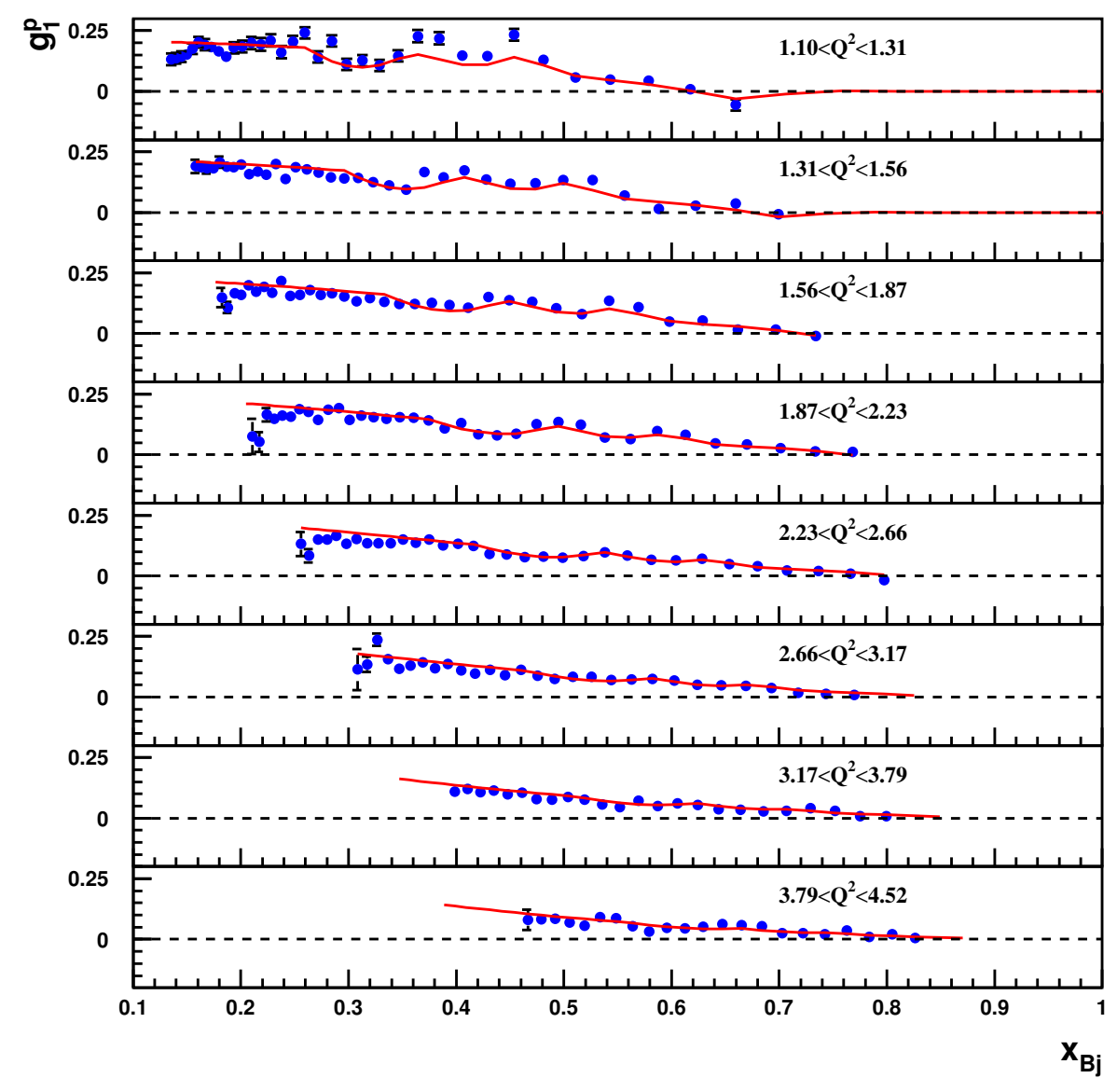

Figure 12: $g_{1}(x)$ vs. $\mathrm{x}$ for the proton, for $\mathrm{E}=5.7 \mathrm{GeV}$ (EG1b). The solid line is a calculation using the parametrization described in the text. Errors are statistical only. 


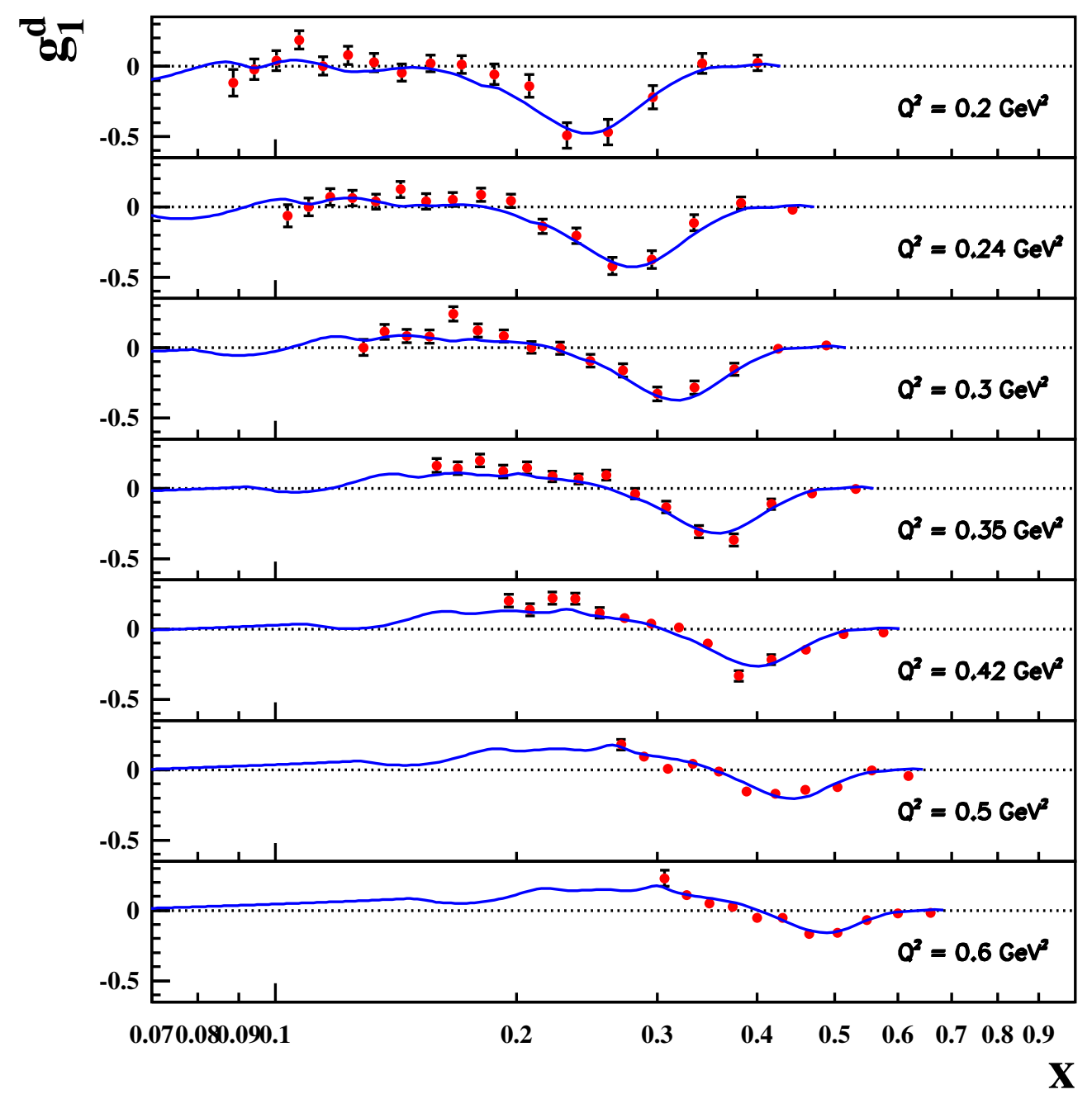

Figure 13: $g_{1}(x)$ vs. $\mathrm{x}$ for the deuteron, for $\mathrm{E}=1.6 \mathrm{GeV}$ (EG1b). The solid line is a calculation using the parametrization described in the text. Errors are statistical only. 


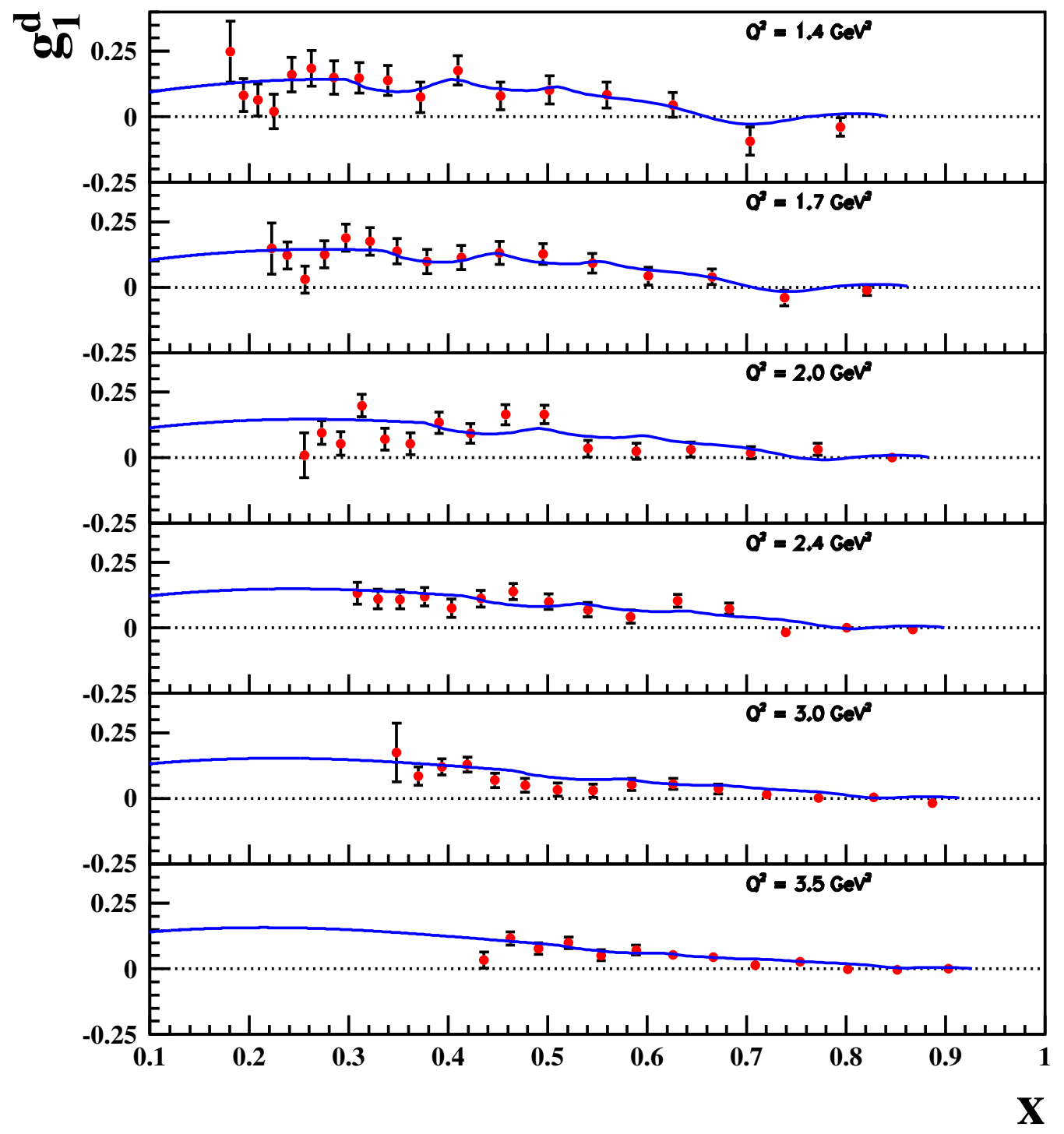

Figure 14: $g_{1}(x)$ vs. $\mathrm{x}$ for the deuteron, for $\mathrm{E}=5.7 \mathrm{GeV}$ (EG1b). The solid line is a calculation using the parametrization described in the text. Errors are statistical only. 


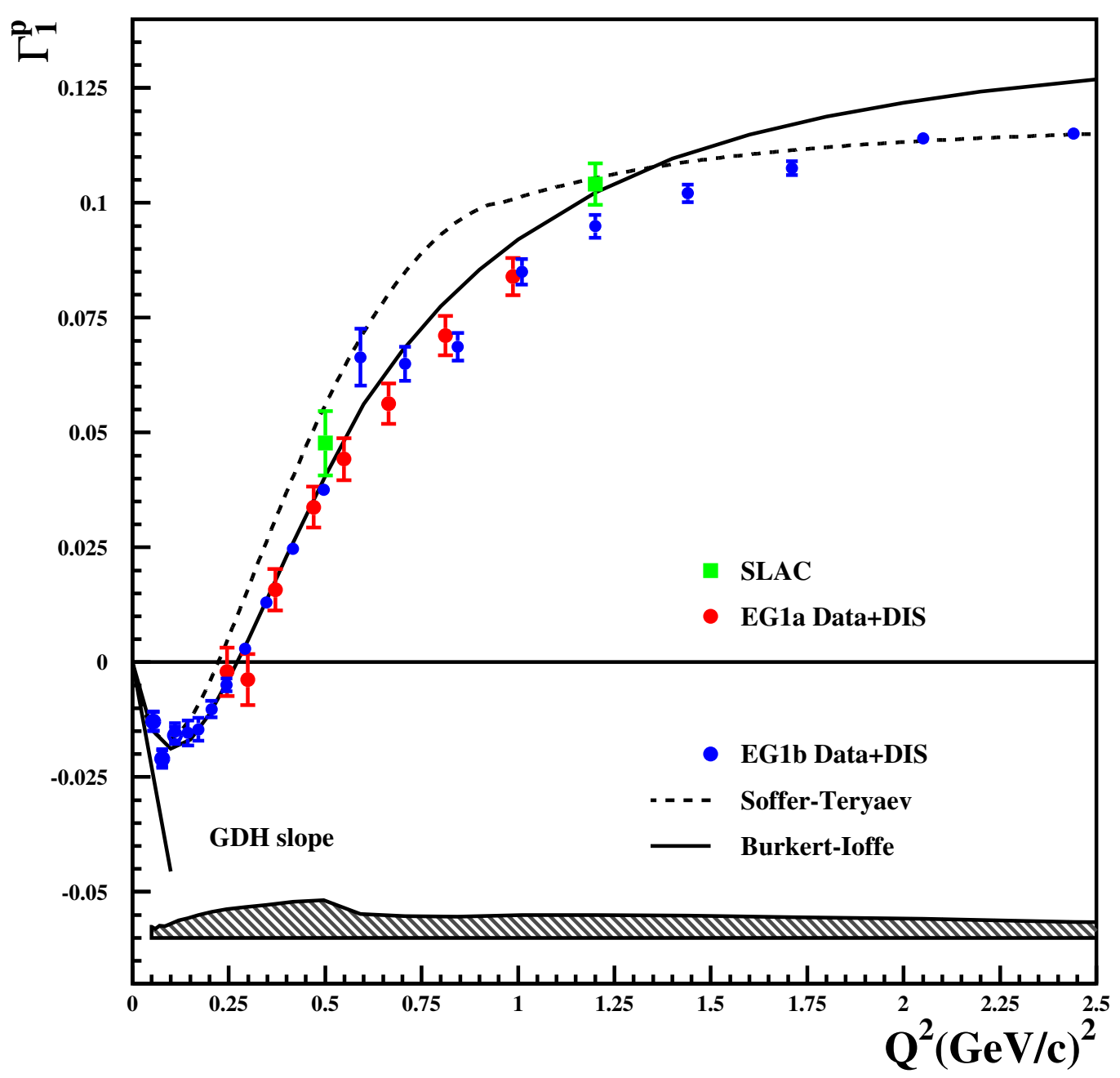

Figure 15: $\Gamma_{1}(x)$ vs. $Q^{2}$ for the proton (EG1b). 


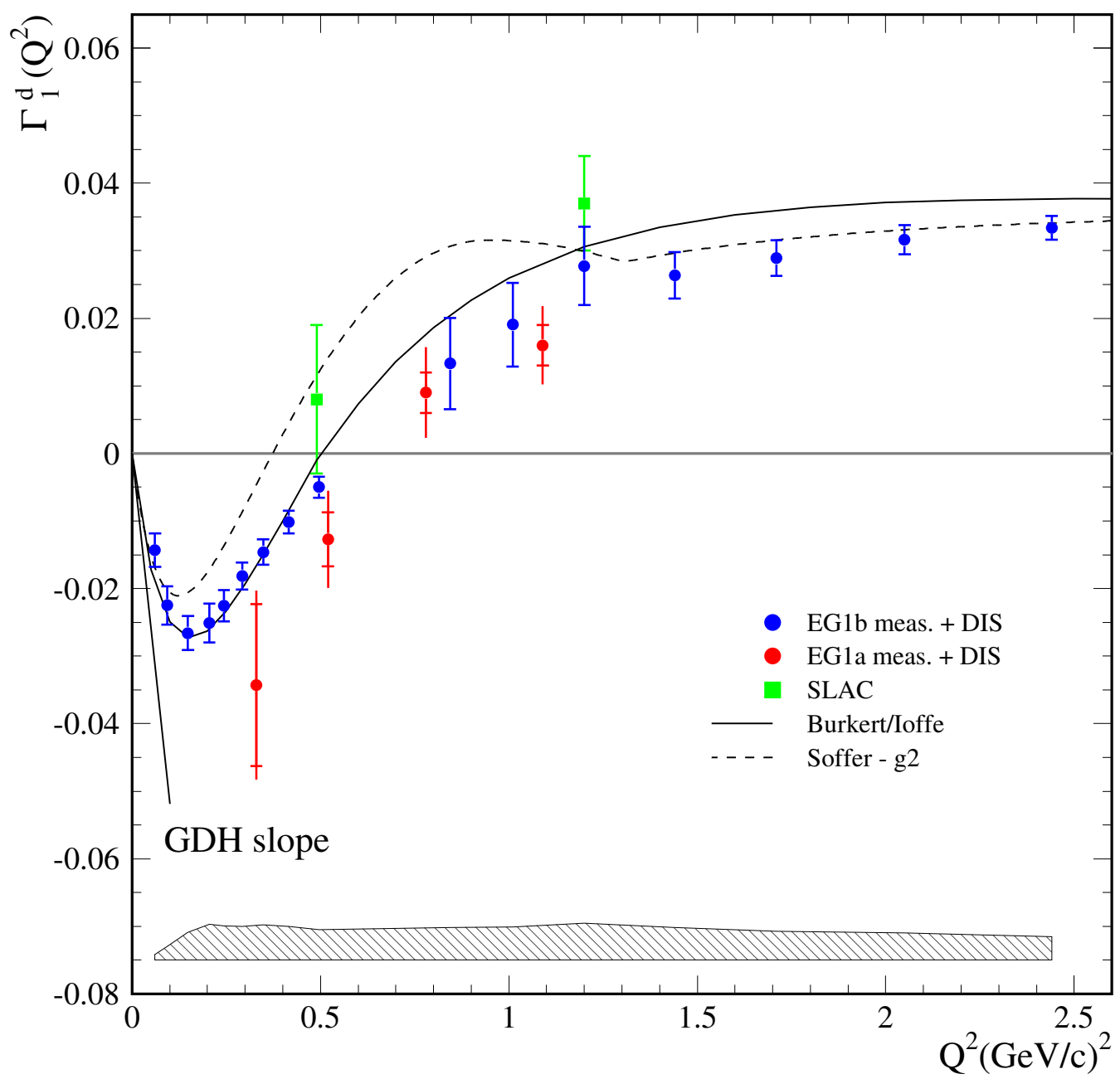

Figure 16: $\Gamma_{1}(x)$ vs. $Q^{2}$ for the deuteron (EG1b). 


\section{$g_{1}^{p} / F_{1}^{p}$ vs $Q^{2}$ (preliminary)}

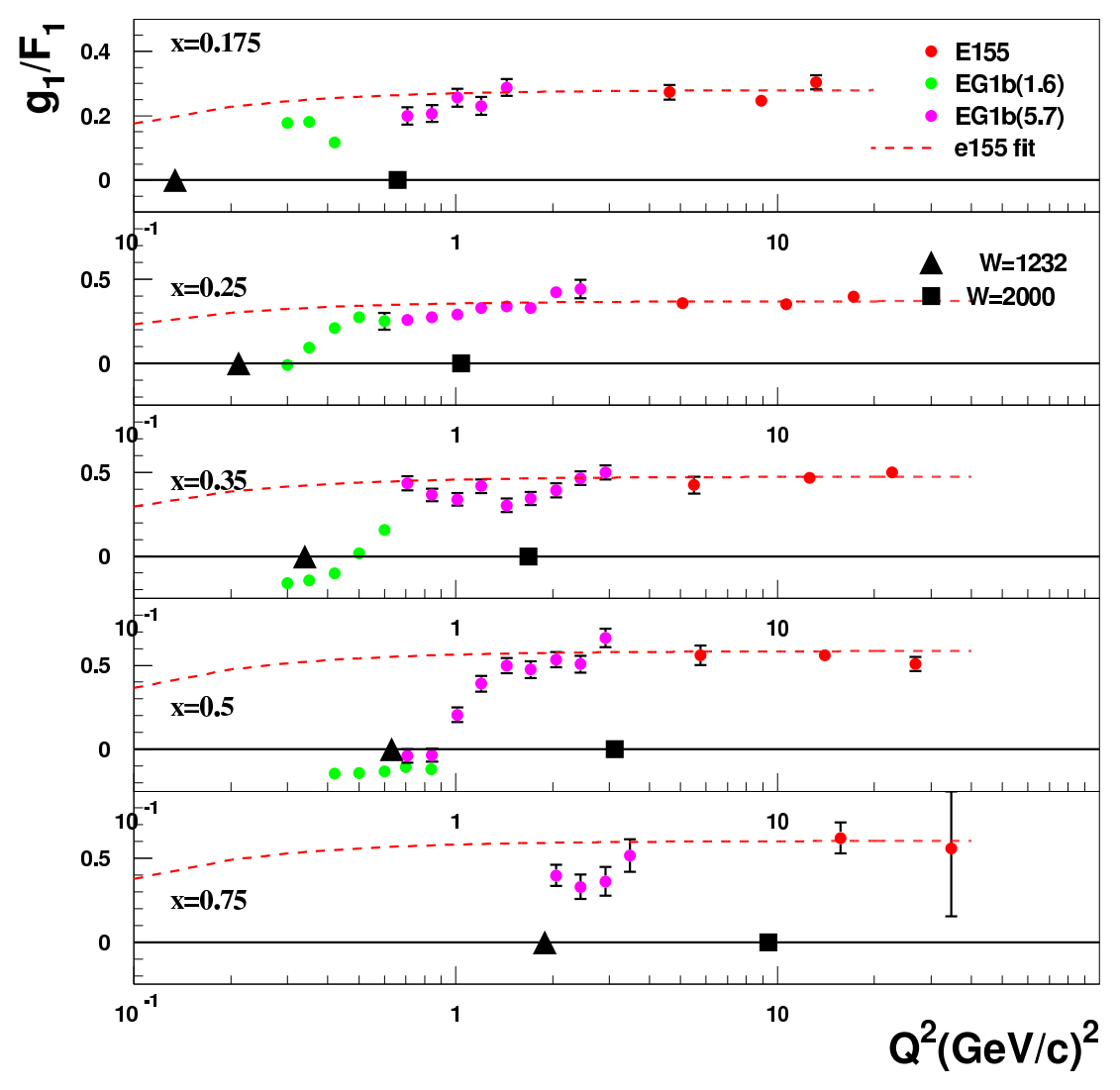

Figure 17: $g_{1}^{p} / F_{1}^{p}$ vs $\mathrm{Q}^{2}$ (EG1b). 


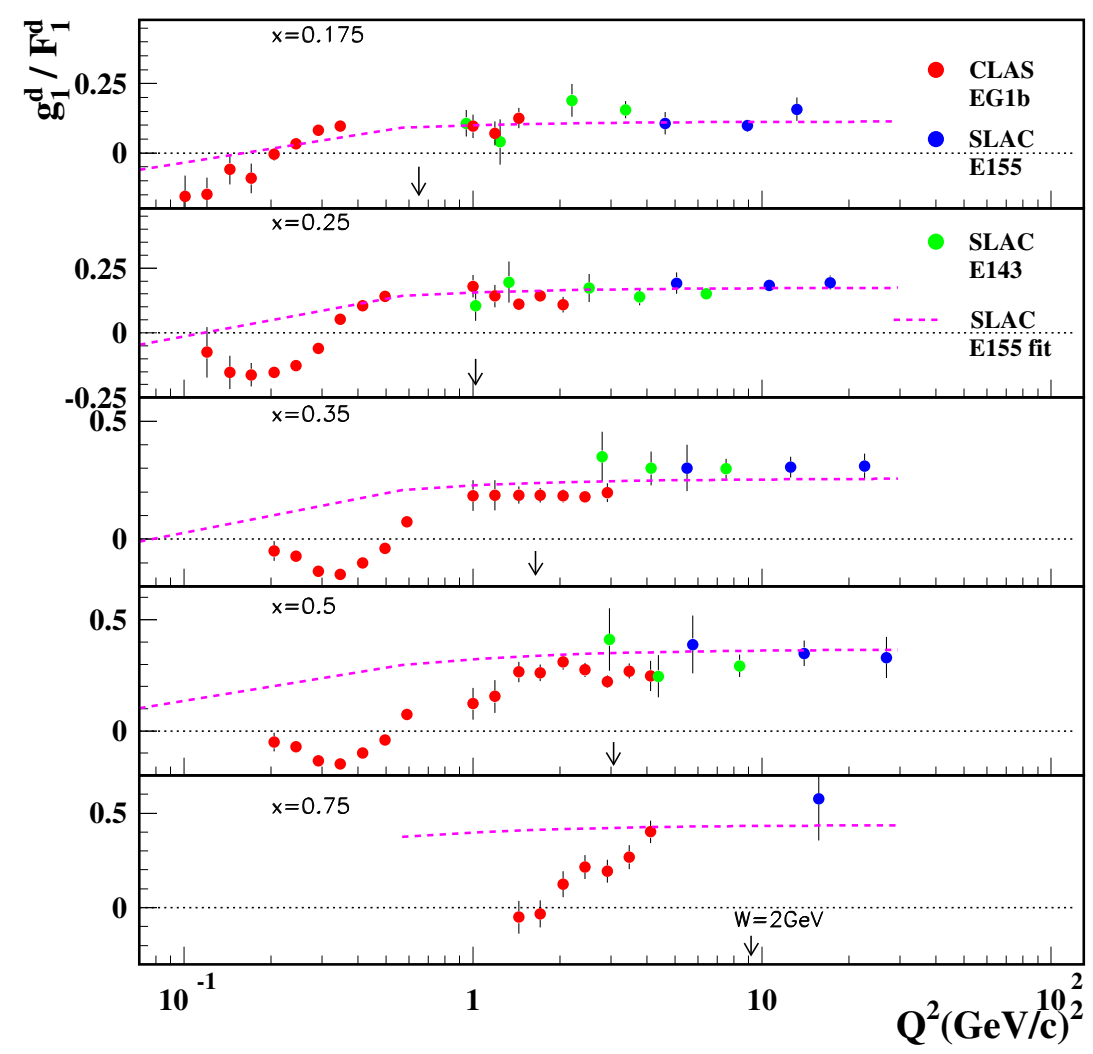

Figure 18: $g_{1}^{p} / F_{1}^{p}$ vs $\mathrm{Q}^{2}(\mathrm{EG} 1 \mathrm{~b})$. 


\section{Bloom-Gilman Duality}

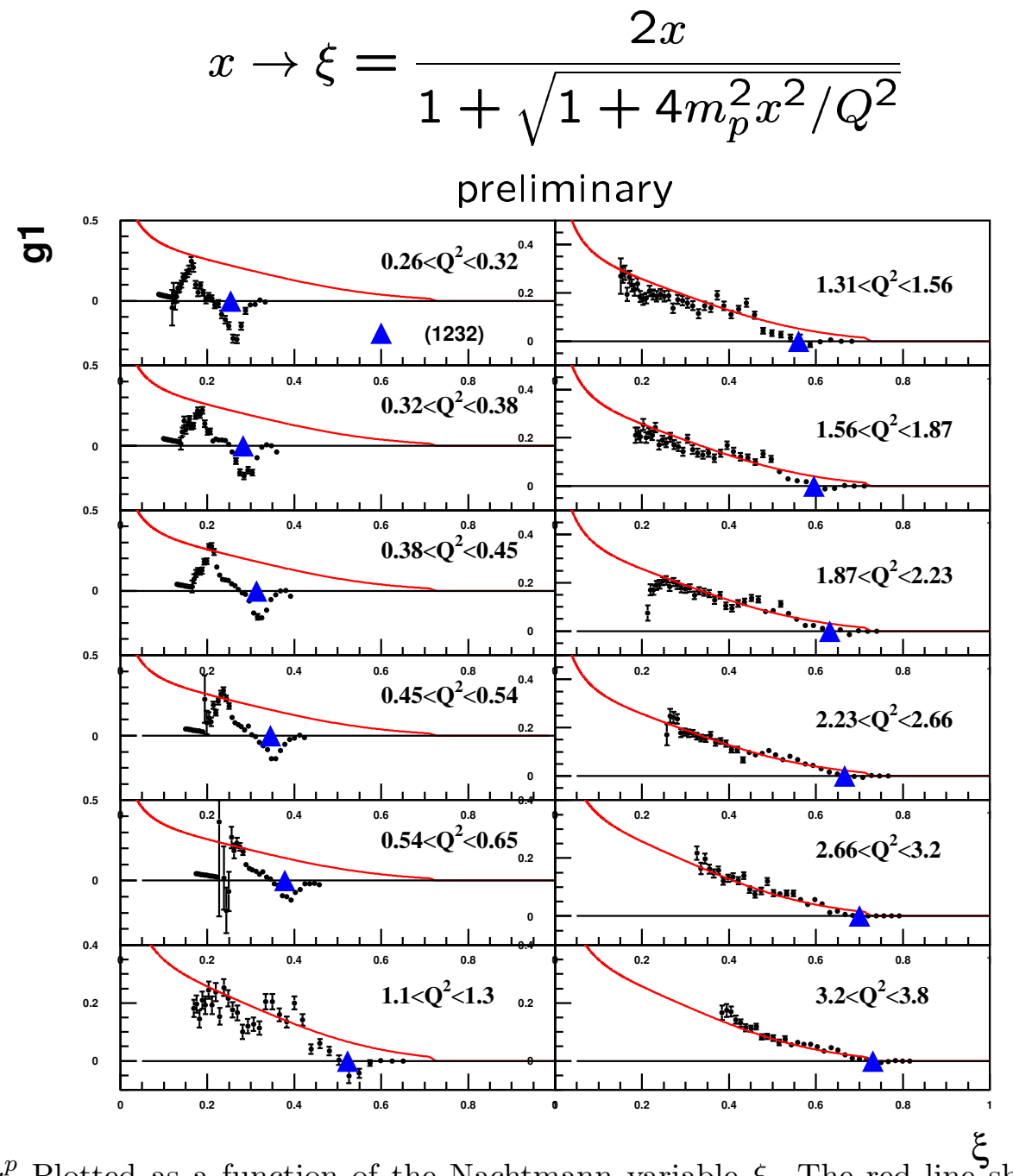

Figure 19: $g_{1}^{p}$ Plotted as a function of the Nachtmann variable $\xi$. The red line shows $g_{1}$ evolved from DIS experiments to $\mathrm{Q}^{2}=10 \mathrm{GeV}^{2}$. 


\section{Asymmetry $A_{1}^{p}\left(x, Q^{2}\right)$}

- $\mathrm{pQCD}: A_{1}^{p}(x \rightarrow 1)=1$

- $\mathrm{SU}(6): A_{1}^{p}=5 / 9$

- EG1b: $\mathrm{W}>1.8 \mathrm{GeV}$

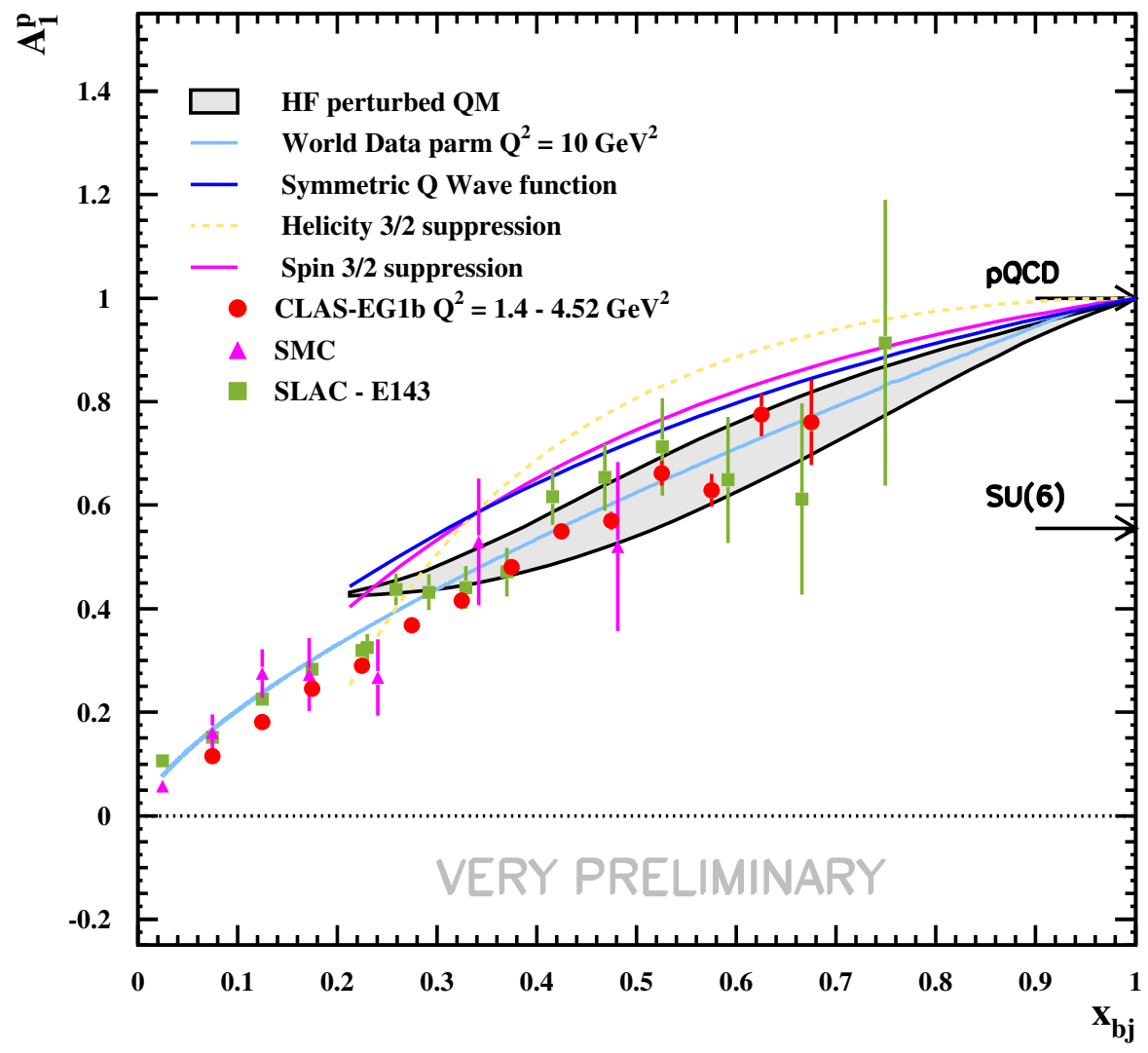

Figure 20: $A_{1}^{p}$ vs. $x_{B j}$. High $\mathrm{Q}^{2}$ CLAS data for $W>1.8 \mathrm{GeV}$ is shown along with data from SMC and SLAC Exp. E143. Parameterizations of the world data and predictions of some representative models are shown. 


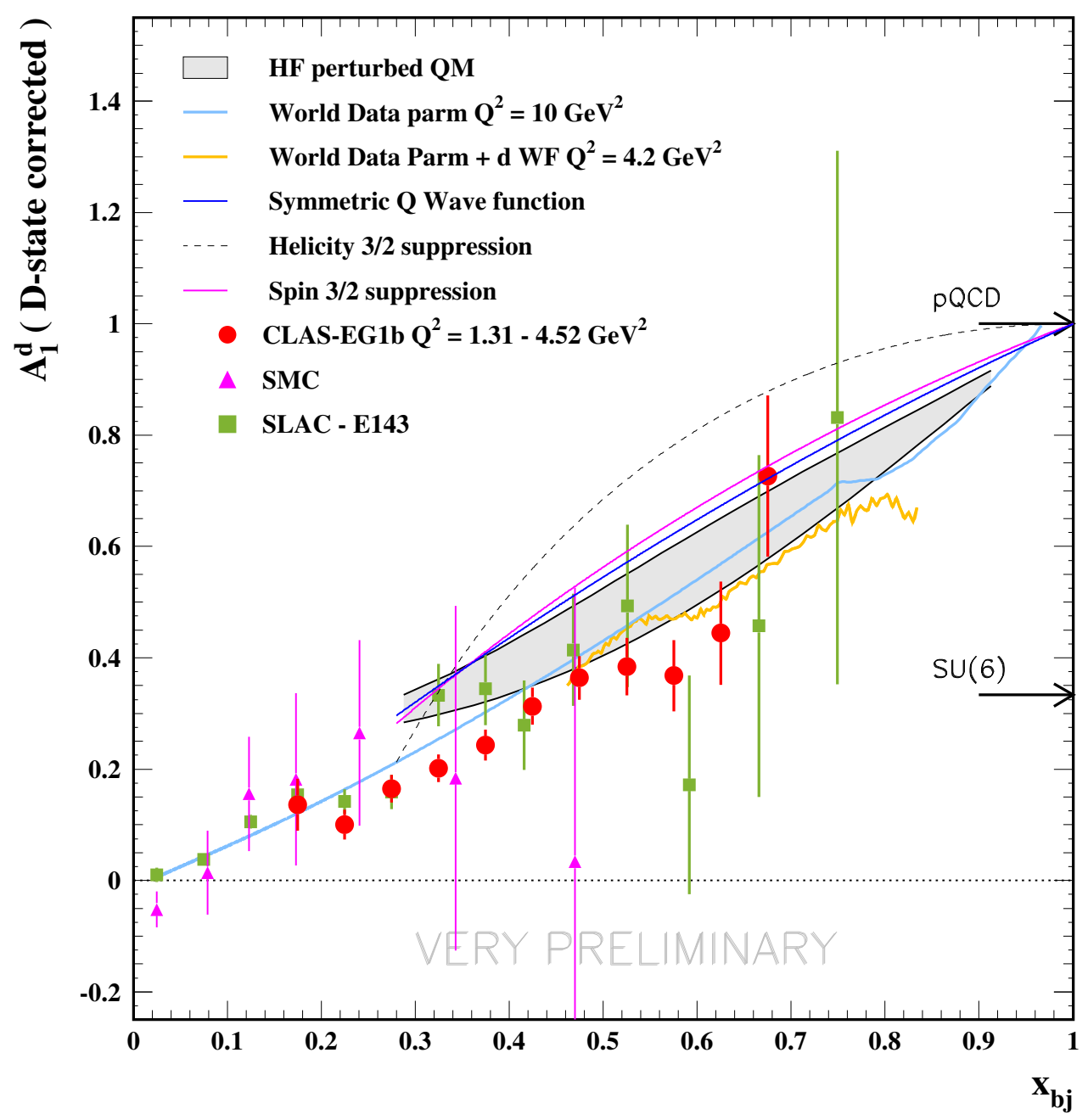

Figure 21: $A_{1}^{d}$ vs. $x_{B j}$. High $\mathrm{Q}^{2}$ CLAS data for $W>1.8 \mathrm{GeV}$ is shown along with data from SMC and SLAC Exp. E143. Parameterizations of the world data and predictions of some representative models are shown. 


\section{PRIMEX Experiment}

R.C. Minehart, Cole Smith, W. Stephens.

R. Minehart is a member of the JLab PRIMEX collaboration led by Ashot Gasparian, Rory Miskimen and Dan Dale. Bill Stephens from our group, making use of expertise acquired through his work on the electronics for the CLAS calorimeter and Cerenkov counters, designed and built specialized electronic summing modules and pulse splitters. R. Minehart also participated in the planning meetings and in the data taking run in the fall of 2004 . The data are now being analyzed by the group, and first results were reported at the April, 2007, meeting of the APS.

\section{A PRECISE MEASUREMENT OF THE $\pi^{+} \rightarrow \pi^{0} e^{+} \nu$ DE- CAY RATE}

PSI Exp. R-89-01.1. The PIBETA Collaboration (U. Virginia, PSI, Swierk, Arizona State U., Dubna, Tbilisi, IRB Zagreb), D. Pocanic(Spokesman).

R.C. Minehart, Cole Smith, W. Stephens.

\subsection{Overview}

Since its inception with a letter of intent to PSI in 1989, we contributed substantially to an experiment at the Paul Scherrer Institute (PSI) in Switzerland to measure the branching ratio for pion beta decay $\left(\pi^{+} \rightarrow \pi^{0}+e^{+}+\nu\right)$. The branching ratio is very small (about $10^{-8}$ ), and at the time of our proposal the best experimental value, obtained by McFarlane et al. [28], was uncertain to $\sim 4 \%$, more than an order of magnitude greater than the theoretical uncertainty of $<0.15 \%[29,30]$. Our goal was to reduce the experimental uncertainty to the order $0.3 \%$, making it comparable to the precision of the measured neutron decay lifetime.

McFarlane et al. measured the decay of pions in flight in order to reduce severe normalization difficulties associated with the decay of stopped pions. However, at the time of our proposal we believed that extensive advances in experimental techniques permitted the use of stopped pions. The ring accelerator at the PSI provided a high pion stopping rate with a favorable time distribution. We therefore designed an apparatus that would use the decay of stopped pions to reduce the error to the order of $0.5 \%$.

The experimental signature of the pion beta decay, $\pi^{+} \rightarrow \pi^{0} e^{+} \nu$, is determined by the prompt decay, $\pi^{0} \rightarrow \gamma \gamma$. The small branching ratio $\left(\simeq 1 \times 10^{-8}\right)$ for the pion beta decay and the required high measurement precision imposed stringent requirements on the experimental apparatus. A large acceptance (nearly $4 \pi$ ) CsI detector was designed to measure with high spatial and energy precision the two photons from the decay of the $\pi^{0}$ produced in beta decay of $\pi+$ mesons stopped in an active target. The decay $\pi^{+} \rightarrow e^{+} \nu$, which yields positrons of energy close to that of the two gamma rays emitted from the $\pi^{0}$ from beta decay, is the normalization reaction. The small branching ratio $\left(\simeq 1 \times 10^{-8}\right)$ for the pion beta decay and the required high measurement precision imposed stringent requirements on the experimental apparatus. A detailed report on the apparatus was published in 2004 [31]. 


\subsection{Results}

Following several years of extensive commissioning, revisions and apparatuscheck-out work, the data taking was completed at the end of 2001, when a total of 70,000 pi-beta decay events had been accumulated. The sensitivity of the apparatus to a variety of pion and muon decay modes provided an important check on the self-consistency of the analysis and of the experiment simulation.

The quality of the data and the yield were in good agreement with the original design. Both the energy distribution and the opening angle distributions for the two photons were in excellent agreement with the simulations. The data rate was about $3.4 \times 10^{-9} \pi \beta$ events per pion stopped in the target, in good agreement with the original proposal, when allowance is made for the data acquisition live time of 0.87 .

The results for the pi-beta branching ratio were published in 2003 [32]. The measured branching ratio is:

$$
\mathrm{BR} \approx\left(1.036 \pm 0.004 \text { (stat.) } \pm 0.005 \text { (syst.) } \times 10^{-8}\right.
$$

where the first uncertainty is the statistical uncertainty, the second is the experimental systematic uncertainty, including the uncertainty in the measurement of the $\pi_{e 2}$ branching ratio. This result can be compared to the following:

- McFarlane et al.: $\mathrm{BR}=(1.026 \pm 0.039) \times 10^{-8}$

- Standard Model (Particle Data Group, 2000): BR=1.038 - $1.041 \times 10^{-8}$, (90\% C.L.)

- Standard Model without radiative corrections: $\mathrm{BR}=1.005-1.007 \times 10^{-8}$

- CVC and Fermi Nuclear Beta Decays (Particle Data Group, 2000): BR = $1.037 \pm$ $0.002 \times 10^{-8}$

We obtained a substantial improvement over the measurement of McFarlane et al., for which the accuracy was too low to distinguish between the calculations with and without radiative correction.

As part of the experiment we also recorded an extensive data set on the radiative decays: $\pi^{+} \rightarrow e^{+} \nu \gamma$ and $\mu^{+} \rightarrow e^{+} \nu \bar{\nu} \gamma$. The first reaction is an important background to the pion beta decay. As reported in another paper published in 2004 [33], our analysis of the radiative pion decay shows a significant departure from Standard Model (SM) predictions in the (high $E_{\gamma}$-low $E_{e}$ ) kinematical region. Detailed cross checks showed that an experimental inefficiency was an improbable explanation. An alternative explanation might be an inadequacy in the $V-A$ description of the pion decay and its radiative corrections, or by an anomalous contribution to the weak interaction. Additional tests to check for experimental sources made at PSI in the summer of 2004, however, were in good agreement with the standard model. 


\section{References}

[1] H. Egiyan, Ph.D thesis, William and Mary (2001).

[2] K. Joo, L.C. Smith et al., Phys. Rev. Lett, 88 (2002) 122001.

[3] K. Joo, L.C. Smith et al., Phys. Rev. C, 68 (2003) 032201.

[4] K. Joo, L.C. Smith et al., Phys. Rev. C, 70 (2004) 042201.

[5] K. Joo, L.C. Smith et al., in preparation (2005).

[6] I. Aznauryan, V. Burkert, H. Egiyan, K. Joo, R.C. Minehart and L.C. Smith, Phys. Rev. C, 71 (2005) 015201.

[7] C. Alexandrou et al., Phys. Rev. Lett. 94 (2005) 021601.

[8] A. Idilbi, X. Ji, J. Ma, Phys. Rev. C, 69 (2004) 014006.

[9] B.W. Filippone and Ziangdong Ji, hep-ph/01001224(2001).

[10] J.D. Bjorken, Phys. Rev. 179, 1547 (1969).

[11] S. Gerasimov, Yad. Fiz. 2, 598(1965), S.D. Drell and A.C. Hearn,Phys. Rev. Lett.16, 908 (1966).

[12] J. Ahrens et al. hep-ex/0105089 (2001).

[13] G. Anton, "Few Body Systems", 177, (1998), Proceedings of the Workshop on N* Physics and Nonperturbative QCD, ed. S. Simula, B. Saghai, N. Mukhopadhyay, V. Burkert, Trento, Italy.

[14] X. Ji, J. Osborne, J. Phys. G27, 127(2001).

[15] V. Bernard et al., arXiv Preprint, hep-ph/0212033.

[16] V. Burkert and Zh. Li, Phys. Rev.,D47, 46 (1993).

[17] V. Burkert and B. Ioffe, Phys. Lett. B296, 223 (1992), J. Exp. Theor. Phys.78, 619 (1994).

[18] V. Burkert, Czech. Journal of Physics, 46, 627 (1996).

41, 205 (1969).

[19] SLAC Radiative program.

[20] AO Program, V.D. Burkert, unpublished.

[21] D. Drechsel, O. Hanstein, S.S. Kamalov, and L. Tiator,Nucl. Phys.A645, 145 (1999).

[22] Xiang-Dong Ji, Chung-Wen Kao, J. Osborne, Phys. Lett. B472,1 (2000).

[23] J. Soffer and O.V. Teryaev, Phys. Rev. Lett.70, 3372 (1993), Phys. Rev.D51, 25 (1995). 
[24] S.A. Larin and J.A.M. Vermaseren, Phys. Lett. B259, 345 (1991).

[25] N. Isgur, Phys. Rev. D58, 034013 (1999).

[26] "Measurement of the Proton Spin Structure Function $g_{1}\left(x, Q^{2}\right)$ for $Q^{2}$ from 0.15 to 1.6 $\mathrm{GeV}^{2}$ with CLAS", R. Fatemi et al. (The CLAS Collaboration), Phys. Rev. Lett. 91, 222002 (2003).

[27] "Measurement of Inclusive Spin Structure Fuctions of the Deuteron with CLAS", J. Yun, et al. (The CLAS Collaboration), Phys. Rev. C67,055204 (2003)

[28] W.K. McFarlane, L.B. Auerbach, F.C. Gaille, V.L. Highland, E. Jastrzembski, R.J. Macek, F.H. Cverna, C.M. Hoffman, G.E. Hogan, R.E. Morgado and R.D. Werbeck, Phys. Rev. D 32, 547 (1985).

[29] A. Sirlin, Phys. Rev. D 35, 3423 (1987), W.J. Marciano and A. Sirlin, Phys. Rev. Lett. 56, 22 (1986).

[30] A. Sirlin, 1989, private communication.

[31] Design, Commissioning and Performance of the PIBETA Detector at PSI", E. Frlez et al. (The PIBETA Collaboration), Nucl. Instrum. Meth. A526, 300 (2004).

[32] "Precise Measurement of the $\pi^{+} \rightarrow e^{+}+\nu$ Branching Ratio", D. Pocanic et al. (The PIBETA Collaboration), Phys. Rev. Lett. 93, 181803 (2004).

[33] "Precise Measurement of the pion axial form-factor in the $\pi^{+} \rightarrow e^{+}+\nu+\gamma$ Decay", E. Frlez et al. (The PIBETA Collaboration), Phys. Rev. Lett. 93, 181804 ) (2004).

[34] "Measurement of the $N \rightarrow \Delta^{+}(1232)$ Transition at High-Momentum Transfer by $\pi^{0}$ Electroproduction", M. Ungaro, P. Stoler, I. Aznauryan, V. D. Burkert, K. Joo, L. C. Smith, (The CLAS Collaboration), Phys. Rev. Lett. 97, 112003 (2006). 


\section{$5 \quad$ Publications and Talks}

\subsection{Published Papers:}

Pion Production:

1. " $\mathrm{Q}^{2}$ Dependence of of Quadrupole Strength in $\gamma^{*} p \rightarrow \Delta^{+}(1232) \rightarrow p \pi^{0}$ Transition", K. Joo et al. (The CLAS Collaboration), Phys. Rev. Lett. 88, 122001 (2002).

2. "First Measurement of the Double Spin Asymmetry in $\vec{e} \vec{p} \rightarrow e^{\prime} \pi^{+} n$ in the Resonance Region", R. DeVita et al. (The CLAS Collaboration), Phys. Rev. Lett. 88, 083001 (2002).

3. "Measurement of the Polarized Structure Function $\sigma_{L T^{\prime}}$ for $p\left(\vec{e}, e^{\prime} p\right) \pi^{o}$ in the $\Delta(1232)$ Resonance Region", K. Joo et al. (The CLAS Collaboration), Phys. Rev. C68 (2003) 032201.

4. "Study of $e p \rightarrow e p \pi^{0}$ in the $\Delta(1232)$ Mass Region using Polarization Asymmetries", A. Biselli et al., Phys. Rev. C68, 035202 (2003).

5. "Measurement of the Polarized Structure Function $\sigma_{L T}^{\prime}$ for $p\left(\vec{e} p, e^{\prime} \pi^{+}\right) n$ in the Delta resonance region", K. Joo et al. (The CLAS Collaboration), Phys. Rev. C70, 042201(R) (2004).

6. "Electroexcitation of the $P_{33}(1232), P_{11}(1440), D_{13}(1520), S_{11}(1535)$ at $\mathrm{Q}^{2}=0.4$ and $0.65(\mathrm{GeV} / \mathrm{c})^{2}$ ", I.G. Aznauryan, et al., Phys. Rev. C, (2004).

7. "Measurement of the Polarized Structure Function $\sigma_{L T^{\prime}}$ for Pion Electroproduction in the Roper Resonance Region", K. Joo et al., (The CLAS Collaboration), Phys.Rev. C72, 058202 (2005).

8. "Single pi + Electroproduction on the Proton in the First and Second Resonance Regions at $0.25 \mathrm{GeV}^{2}<Q^{2}<0.65 \mathrm{GeV}^{2}$ Using CLAS", H. Egiyan, I.G. Aznauryan, V.D. Burkert, K.A. Griffioen, K. Joo, R. Minehart, L.C. Smith, (The CLAS Collaboration), Phys.Rev. C73, 025204 (2006).

9. " Measurement of the $N \rightarrow \Delta^{+}(1232)$ Transition at High-Momentum Transfer by $\pi^{0}$ Electroproduction", M. Ungaro, P. Stoler, I. Aznauryan,V. D. Burkert, K. Joo, L. C. Smith, (The CLAS Collaboration), Phys. Rev. Lett. 97, 112003 (2006).

Spin Structure Functions:

1. "Measurement of the Proton Spin Structure Function $g_{1}\left(x, Q^{2}\right)$ for $Q^{2}$ from 0.15 to 1.6 $\mathrm{GeV}^{2}$ with CLAS", R. Fatemi et al. (The CLAS Collaboration), Phys. Rev. Lett. 91, 222002 (2003).

2. "Measurement of Inclusive Spin Structure Fuctions of the Deuteron with CLAS", J. Yun, et al. (The CLAS Collaboration), Phys. Rev. C67,055204 (2003) 
3. "A Complete Measurement of the $F_{2}$ Proton Structure Function in the Resonance Region and the Evaluation of the Moments", M. Osipenko, et al. (The CLAS Collaboration) Phys. Rev., D 67, 092001 (2003)

4. "Measurement of the Spin Structure Functions in the Resonance Region for $\mathrm{Q}^{2}$ from 0.15 to $1.6 \mathrm{GeV}^{2}$ ", R. Fatemi et al. (The CLAS Collaboration), Phys. Rev. Lett. 91. $222002(2003)$.

5. "Protons and neutron spin structure functions in and near the resonance region.", R.C. Minehart (for the CLAS Collaboration), Presented at Intersections of particle and nuclear physics, New York, May, 2003, pub, in AIP Conf. Proc. 698, 632 (2004).

6. "Experimental Determination of the Evolution of the Bjorken Integral at Low $\mathrm{Q}^{2}$ ", A. Deur et al. Phys. Rev. Lett. 93, 212001 (2004).

7. "Measurement of he Deuteron structure function $F_{2}$ in the resonance region and evaluation of its moments", M. Osipenko et al. (CLAS Collaboration), Phys. Rev. C73, 045205 (2006).

8. "Measurement of the $x$ - and $\mathrm{Q}^{2}$-dependence of the asymmetry $A_{1}$ on the nucleon", K.V. Dharmawardane et al. (CLAS Collaboration), Phys. Lett. B641, 11-17 (2006).

9. "Quark-hadron duality in spin structure functions $\mathrm{g}(1) \mathrm{p}$ and $\mathrm{g}(1) \mathrm{d}$ ", P.E. Bosted et al. (The CLAS Collaboration). Phys. Rev. C75, 035203 (2007).

\section{PiBeta Papers:}

1. Design, Commissioning and Performance of the PIBETA Detector at PSI", E. Frlez et al. (The PIBETA Collaboration), Nucl. Instrum. Meth. A526, 300 (2004).

2. "Precise Measurement of the $\pi^{+} \rightarrow e^{+}+\nu$ Branching Ratio", D. Pocanic et al. (The PIBETA Collaboration), Phys. Rev. Lett. 93, 181803 (2004).

3. "Precise Measurement of the pion axial form-factor in the $\pi^{+} \rightarrow e^{+}+\nu+\gamma$ Decay", E. Frlez et al. (The PIBETA Collaboration), Phys. Rev. Lett. 93, 181804 ) (2004). 
Other CLAS papers:

1. " $\eta$ Photoproduction on the Proton for Photon Energies from 0.75 to $1.95 \mathrm{GeV}$ ", M. Dugger et al. (The CLAS Collaboration), Phys. Rev. Lett. 89, 222002 (2002).

2. "Observation of an Exotic $\mathrm{S}=+1$ Baryon in Exclusive Photoproduction from the Deuteron", S. Stepanyan et al., Phys. Rev. Lett. 91, 252001 (2003).

3. "Observation of nuclear scaling in the $A(e, e)$ reaction at $x_{B}>1$ ", K. Sh. Egiyan et al. (The CLAS Collaboration), Phys. Rev. C68, 014313 (2003).

4. "Measurement of $e p \rightarrow e^{\prime} p \pi^{+} \pi^{-}$and Baryon Resonance Analysis", M. Ripani, et al. (The CLAS Collaboration), Phys. Rev. Lett. 91, 022002, (2003).

5. "The CEBAF Large Acceptance Spectrometer", B. Mecking, et al. (The CLAS Collaboration). Nucl. Inst. and Meth. 503/3, 513 (2003)

6. "First Measurement of Transferred Polarization in the Exclusive $\vec{e} p \rightarrow e^{\prime} K^{+} \vec{\Lambda}$ Reaction", D. Carman, et al.(The CLAS Collaboration), Phys. Rev. Lett. 90, 131804 (2003).

7. "Photoproduction of the $\omega$ Meson on the Proton at Large Mementum Transfer", M. Battaglieri, et al. (The CLAS Collaboration), Phys. Rev. Lett. 90, 022002 (2003).

8. "A Kinematically Complete Measurement of the $F_{2}$ Proton Structure Function in the Resonance Region and the Evaluation of the Moments", M. Osipenko et al. (The CLAS Collaboration), Phys. Rev., D 67, 092001 (2003)

9. "Complete measurement of three-body photodisintegration of ${ }^{3} \mathrm{He}$ for photon energies between 0.35 and 1.55 GeV", S. Niccolai et al. (The CLAS Collaboration), Phys. Rev. C70, 064003 (2004).

10. "Observation of an Exotic Baryon with $\mathrm{S}=+1$ in Photoproduction from the Proton", V. Kubarovsky et al. (The CLAS Collaboration), Phys. Rev. Lett. 92, 032001 (2004).

11. "Tensor polarization of the $\phi$ meson photoproduced at high t", K. McCormick et al. (The CLAS Collaboration), Phys. Rev. C69, 032203(R) (2004).

12. "Hyperon photoproduction in the nucleon resonance region", J. W. McNabb et al. (The CLAS Collaboration), Phys. Rev. C69, 042201(R) (2004).

13. "Measurement of Beam-Spin Asymmetries for Deep Inelastic $\pi^{+}$Electroproduction", H. Avakian, et al. (The CLAS Collaboration), Phys. Rev. D69 (2004) 112004.

14. "Observation of an Exotic Baryon with $\mathrm{S}=+1$ in Photoproduction from the Proton", V. Koubarovski et al. (The CLAS Collaboration), Phys. Rev. Lett. 92, 032001 (2004), Erratum: Phys. Rev. Lett. 92, 049902 (2004).

15. "Complete Measurement of the Three-body Photodisintegration of ${ }^{3} \mathrm{He}$ for photon energies between 0.35 and $1.55 \mathrm{GeV}$ ", S. Nicolai, et al. (The CLAS Collaboration), Phys. Rev. C70, 064003 (2004). 
16. "Exclusive $\rho^{0}$ meson electroproduction from hydrogen at CLAS", C. Hadjidakis et al. (The CLAS Collaboration), Phys. Lett. B605, 256 (2005).

17. "Survey of $A_{L T}^{\prime}$ asymmetries in semi-exclusive electron scattering on ${ }^{4} \mathrm{He}$ and ${ }^{12} \mathrm{C}$ ", D. Protopopesecu et al., Nucl. Phys. A748, 357 (2005).

18. "Deeply virtual and exclusive electroproduction of $\omega$ mesons", L. Morand et al., (The CLAS Collaboration), Eur.Phys.J. A24, 445 (2005).

19. "Beam-Helicity Asymmetries in Double-Charged-Pion Photoproduction on the Proton", S. Strauch et al., (The CLAS Collaboration), Phys. Rev. Lett. 95, 162003 $(2005)$.

20. "Differential Cross Sections for $\gamma+p \rightarrow K^{+}+Y$ for $\Lambda$ and $\Sigma^{0}$ Hyperons", R. Bradford et al., (The CLAS Collaboration), Phys.Rev. C73, 035202 (2006).

21. " $\eta$ ' photoproduction on the proton for photon energies from 1.527 to $2.227 \mathrm{GeV}$ ", M. Dugger et al., (The CLAS Collaboration), Phys.Rev.Lett. 96, 062001 (2006).

22. "Search for $\Theta^{+}(1540)$ pentaquark in high statistics measurement of $\gamma p \rightarrow \bar{K}^{0} K^{+} n$ at CLAS", M. Battaglieri et al., (The CLAS Collaboration), Phys.Rev.Lett. 96, 042001 (2006).

23. "Measurement of 2- and 3-Nucleon Short Range Correlation Probabilities in Nuclei", K. S. Egiyan et al., (The CLAS Collaboration), Phys. Rev. Lett. 96 ,082501 (2006).

24. "Electron Scattering From High-Momentum Neutrons in Deuterium", A.V. Klimenko et al., (The CLAS Collaboration), Phys.Rev. C73, 035212 (2006).

\subsection{Talks given at Meetings:}

Pion Production:

1. "E2/M1 and C2/M1 with CLAS", L. C. Smith, N*2002 Conference, Pittsburgh, Oct. 9-12, 2002.

2. "Electroproduction of the (1232) for Q2=0.1-0.3 GeV2 using CLAS", L. C. Smith, APS Meeting, Philadephia, April 5-8, 2003.

3. "CLAS Results on Delta and Roper", L. C. Smith, N*2004, Grenoble, France, March 24-27, 2004.

4. "CLAS Measurement of Pion Electroproduction in the Delta(1232) Region at Q2=0.10.3 GeV2", L.C. Smith, APS April Meeting, May 1-4, 2004.

Spin Structure Functions:

1. "Measurement of the Structure Functions $g_{1}^{p}$ and $g_{1}^{n}$ with CLAS at Jefferson Lab", Y. Prok, N*2002 Conference, Pittsburgh, Oct. 9-12, 2002. 
2. "Measurement of the Structure Functions g1p and g1n with the CLAS at Jefferson Lab", Y. Prok, Coral Gables Conference 2002, Dec. 11-15, 2002.

3. "Measurements of the ep and ed Polarized Structure Functions at Energies of 1.6 and 5.7 GeV Using CLAS', Y. Prok, APS Meeting, Philadephia, April 5-8, 2003.

4. "Spin Program with CLAS at JLab", R. Minehart, SPIN-Praha 2004, Prague, July 5-10, 2004.

5. "Protons and neutron spin structure functions in and near the resonance region.", R.C. Minehart (for the CLAS Collaboration), Presented at Intersections of particle and nuclear physics, New York, May, 2003, pub, in AIP Conf. Proc. 698, 632 (2004). 